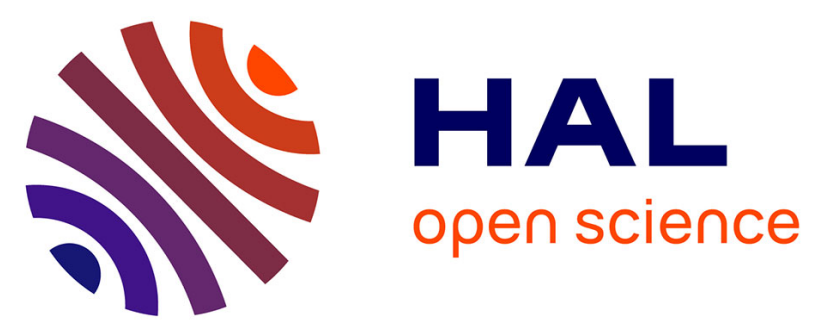

\title{
The ratio processing system and its role in fraction understanding: Evidence from a match-to-sample task in children and adults with and without dyscalculia
}

Parnika Bhatia, Maël Delem, Jessica Léone, Esther Boisin, Anne Cheylus, Marie-Line Gardes, Jérôme Prado

\section{To cite this version:}

Parnika Bhatia, Maël Delem, Jessica Léone, Esther Boisin, Anne Cheylus, et al.. The ratio processing system and its role in fraction understanding: Evidence from a match-to-sample task in children and adults with and without dyscalculia. Quarterly Journal of Experimental Psychology, 2021, 42 (11), pp.3396-3410. 10.1177/1747021820940631 . hal-03052304

\author{
HAL Id: hal-03052304 \\ https://hal.science/hal-03052304
}

Submitted on 5 Oct 2021

HAL is a multi-disciplinary open access archive for the deposit and dissemination of scientific research documents, whether they are published or not. The documents may come from teaching and research institutions in France or abroad, or from public or private research centers.
L'archive ouverte pluridisciplinaire HAL, est destinée au dépôt et à la diffusion de documents scientifiques de niveau recherche, publiés ou non, émanant des établissements d'enseignement et de recherche français ou étrangers, des laboratoires publics ou privés. 


\title{
The ratio processing system and its role in fraction understanding:
}

\section{Evidence from a match-to-sample task in children and adults with and without dyscalculia}

\author{
Parnika Bhatia $^{1}$, Maël Delem ${ }^{1}$, Jessica Léone ${ }^{1}$, Esther Boisin ${ }^{1}$, Anne Cheylus ${ }^{1}$, Marie-Line \\ Gardes $^{1}$ \& Jérôme Prado ${ }^{1}$ \\ ${ }^{1}$ Lyon Neuroscience Research Center (CRNL), Experiential Neuroscience and Mental Training \\ Team, INSERM U1028 - CNRS UMR5292, University of Lyon, Lyon, France.
}

\begin{abstract}
Correspondence should be addressed to P.B. (parnika.bhatia@etu.univ-lyon1.fr) or J.P. (jerome.prado@univ-lyon1.fr), Lyon Neuroscience Research Center, CH Le Vinatier, 95 bd Pinel, 69675 Bron Cedex, France. Phone: +33 (0)4 37911265
\end{abstract}

Declarations of interest: none

Word count (text plus references): 13,844

Funding: This research was supported by a grant from the Région Auvergne Rhône Alpes (Pack Ambition Recherche Dyscog) to M-L.G. and J.P.

\begin{abstract}
Acknowledgments: We thank Percival G. Matthews and Edward M. Hubbard for helpful discussions prior to performing these experiments.
\end{abstract}




\begin{abstract}
It has been hypothesized that the human neurocognitive architecture may include a perceptual Ratio Processing System (RPS) that supports symbolic fraction understanding. In the present study, we aimed to provide further evidence for the existence of the RPS by exploring whether individuals with a range of math skills are indeed perceptually sensitive to non-symbolic ratio magnitudes. We also aimed to test to what extent the RPS may underlie symbolic fraction processing in those individuals. In a match-to-sample task, typical adults, elementary school children, and adults with dyscalculia were asked to match a non-symbolic ratio (i.e., target) to one of two non-symbolic ratios (i.e., the match and distractor). We found that all groups of participants were sensitive to the ratio between the match and the distractor, suggesting a common reliance on the RPS. This ratio sensitivity was also observed in another group of typical adults who had to choose which of two symbolic fractions match a non-symbolic ratio, indicating that the RPS may also contribute to symbolic fraction understanding. However, no ratio dependence was observed when participants had to choose which of two symbolic fractions match another symbolic fraction, suggesting that reliance on the RPS in symbolic fraction processing is limited and may not support exact fraction processing.
\end{abstract}

Keywords: Ratio Processing System, Symbolic Fractions, Match to Sample task, Dyscalculia 


\section{RPS AND SYMBOLIC FRACTIONS}

\section{Introduction}

Fractions have a key role to play in the holistic development of numerical concepts. From a life skills perspective, they are crucial for calculating discounts, managing medical dosage, cooking, banking, and in laying the foundation for careers in science, technology, engineering, and mathematics. From an educational perspective, they are central in mathematics curriculum at both elementary and secondary school level and have a strong predictive relationship with algebra and mathematics achievement later in life (Bailey, Hoard, Nugent, \& Geary, 2012; Booth \& Newton, 2012; Siegler et al., 2012). Although children receive fraction instruction beginning around 8 years of age, they continue to struggle with fractions in high school and even after college (Kloosterman, 2010; Stigler, Givvin, \& Thompson, 2010; Van Hoof, Lijnen, Verschaffel, \& Van Dooren, 2013). Attesting to the problem, mathematic educators and researchers worldwide have noted a pervasive lack of fraction understanding among students (Chan, Leu, \& Chen, 2007; Ni, 2001; Yoshida \& Sawano, 2002).

Why are fractions difficult?

Research in mathematics education and cognitive science has documented two main reasons that may explain why adults and children across countries face difficulties with fractions. The first reason is that accessing the holistic magnitude of symbolic fractions appears to be difficult for most individuals (Kallai \& Tzelgov, 2009, 2012; Stafylidou \& Vosniadou, 2004). Being able to estimate the magnitude of fractions is critical in the development of conceptual knowledge of fractions and predicts success in fraction computation and arithmetic (Siegler \& Pyke, 2013; Siegler, Thompson, \& Schneider, 2011). Unfortunately, the challenges to access and 
process the holistic magnitude of fractions are all too common even in expert mathematicians (Obersteiner, Van Dooren, Van Hoof, \& Verschaffel, 2013).

Limited understanding of fraction magnitude is quite evident in children. For instance, a study on $6^{\text {th }}$ and $8^{\text {th }}$ graders noted that one-third of the students solved fraction arithmetic problems by handling numerators and denominators separately (Siegler \& Pyke, 2013). In a oneto-one instructional session, seven out of eight $6^{\text {th }}$ graders responded $1 / 8$ to be greater than $1 / 6$ (Mack, 1990). It has also been observed that students are challenged by the concepts of equivalence because they fail to understand that different symbolic fractions can represent the same magnitude (e.g. 4/8 and 2/4) (Clarke \& Roche, 2009). Additionally, studies on adult participants suggest that the holistic magnitude of fractions can be accessed when componential strategies relying on the separate processing of numerators and denominators fail (Meert, Grégoire, \& Noël, 2009, 2010; Meert, Grégoire, Seron, \& Noël, 2012).

A second reason for the difficulty individuals face with fractions is that they often assume that properties of whole numbers apply to all numbers (Siegler, Fazio, Bailey, \& Zhou, 2013; Vamvakoussi \& Vosniadou, 2010). This so-called whole-number bias (Braithwaite \& Siegler, 2017; Ni \& Zhou, 2005) leads to the misapplication of whole-number concepts and procedures on fractions. For instance, children might erroneously think that fractions, like whole numbers, have unique successors, can be counted, can be represented by a single symbol, or increase in magnitude if they are multiplied (Siegler et al., 2012). The whole-number bias often leads to erroneous conclusions, such as those reached when performing arithmetic operations separately on the numerators and denominators of the operands (e.g., 1/8 +1/8=2/16) (Mack, 1995; Stigler et al., 2010). For example, when estimating the answer to a problem such as $12 / 13+7 / 8,76 \%$ of 13-year-olds choose either 19 or 21 (i.e., the addition of numerators and denominators separately) 


\section{RPS AND SYMBOLIC FRACTIONS}

(Carpenter, Kepner, Corbitt, Lindquist, \& Reys, 1980). Importantly, this bias is difficult to overcome. A qualitative study on 52 seventh graders demonstrated that students were persistent in their way of reasoning about fractions. This indicates that the most frequent errors were not random but rooted in deep misconceptions (González-Forte, Fernández, \& Dooren, 2019).

Finally, other sources of difficulties with fractions have been noted by researchers on math education. For example, these may stem from a limited understanding of part-whole concepts (Pitkethly and Hunting 1996; Steffe and Olive 2010; Streefland 1991), the multiple ways in which rational numbers can be represented (Kieren, 1981), as well as the variety of procedures that are involved in fraction arithmetic (Siegler et al., 2013). For example, adding fractions with common denominators (e.g., $2 / 6+3 / 6$ ) requires one to maintain the denominator constant and add the numerator, whereas multiplying the same fractions (e.g., $2 / 6 \times 3 / 6$ ) requires one to multiply both the numerator and denominator. Thus, the procedures used in fraction arithmetic are varied and complex.

\section{The Innate Constraint Account}

Owing to the severity of challenges faced, some researchers have argued that the human cognitive architecture is endowed with a foundational system that is exclusively designed to deal with discrete quantities (Gelman \& Meck, 1983; Leslie, Gelman, \& Gallistel, 2008). This Approximate Number System (ANS) is thought to be inherited from evolution because early competence with discrete quantities can be observed in many animal species, including rats, pigeons, monkeys, chimpanzees, and several infant studies (Boysen \& Capaldi, 1993; Dehaene, Dehaene-Lambertz, \& Cohen, 1998a). In humans, the ANS is also thought to serve as a 
foundation for symbolic, whole number concepts (Bailey, Hoard, Nugent, \& Geary, 2012; Dehaene \& Cohen, 2007; Dehaene, Dehaene-Lambertz, \& Cohen, 1998b; Feigenson, Dehaene, \& Spelke, 2004; Piazza, 2010) (but see De Smedt et al., 2013; Leibovich et al., 2017). As this system is not designed to deal with the physical features of fractional and rational number quantities, it has been theorized that it may pose constraints on acquiring rational number concepts (Bonato, Fabbri, Umiltà, \& Zorzi, 2007; Feigenson et al., 2004). According to this Innate Constraint account, it is not surprising that acquiring elementary level skills in wholenumbers is more efficient and easier as compared to fractions and rational numbers. Therefore, the innate mechanism that is assumed to help children learn discrete number concepts may impede and/or interfere with children's acquisition of fractional numbers.

\section{The Ratio Processing System}

However, a growing body of evidence is difficult to reconcile with the Innate Constraint account. For instance, McCrink and Wynn (2007) observed that 6-month old infants, when habituated to specific non-symbolic ratios, tend to look longer at novel ratios (differing by a factor of two), indicating that infants as young as 6 months can process differences between nonsymbolic ratios. By the age of 4, children can also accurately perform addition and subtraction on part-whole representations of fractions (Mix, Levine, \& Huttenlocher, 1999). Moreover, studies on adults have revealed that non-symbolic ratios are encoded by specific neuronal populations in the parietal cortex and lateral prefrontal cortex (Jacob \& Nieder, 2009a, 2009b). Research on non-human primates also shows that trained monkeys can match non-symbolic ratios accurately (Vallentin \& Nieder, 2010). Taken together, these studies raise the possibility that there exists an evolutionary old perceptual system for representing and processing non-symbolic ratios in the 


\section{RPS AND SYMBOLIC FRACTIONS}

human brain. This system, which has been termed the Ratio Processing System (RPS), is thus also likely to be present relatively early in children's development (Lewis, Matthews, \& Hubbard, 2015).

Critically, some have argued that the RPS might provide a basis for processing symbolic fraction magnitudes in humans (Lewis, Matthews, Hubbard, 2016). This is suggested by four lines of evidence. First, individual differences in non-symbolic ratio processing relate to individual differences in symbolic fraction processing in school-aged children (Möhring, Newcombe, Levine, \& Frick, 2016) and adults (Matthews, Lewis, \& Hubbard, 2016). Second, when adults compare the magnitude of symbolic fractions as well as the magnitude of nonsymbolic ratios (e.g., pairs of lines), their performance depends on the ratio between the magnitudes (i.e., a Ratio of Ratios, RoR) (Hurst \& Cordes, 2016; Matthews \& Ziols, 2019) For example, participants are faster and more accurate at comparing symbolic fractions and nonsymbolic ratios when the RoR is large (e.g., $2 / 6$ versus $1 / 9=3: 1$ ) than when it is small (e.g., $4 / 5$ versus $2 / 3=6: 5$ ) (Hurst \& Cordes, 2016). This effect - also observed with whole numbers - is generally thought to indicate that numerical magnitudes are ordered along a mental number line (Moyer \& Landauer, 1967). Accordingly, representations of magnitudes are more difficult to distinguish when the distance between them decreases (i.e., a distance effect) and when the overall magnitude increases (i.e. a size effect). This combined effect of distance and size results in performance that varies with the ratio between magnitudes (in the case of fractions a RoR). Third, a study has found that adults are faster at comparing symbolic fractions to non-symbolic fractions (i.e., a mixed-format comparison) than symbolic fractions to symbolic fractions (i.e., a within-format comparison) (Matthews \& Chesney, 2015). This also suggests that a shared magnitude code might be accessed intuitively when performing mixed-format comparisons (Matthews \& Chesney, 2015; Matthews, Chesney, \& McNeil, 2014). Fourth, fMRI studies have 
shown that processing both symbolic fractions and non-symbolic ratios rely on similar brain regions in and around the intraparietal sulcus (Ischebeck et al., 2009; Jacob \& Nieder, 2009a, 2009b; Mock et al., 2018, 2019). Therefore, both behavioural and neural evidence raise the possibility that a perceptual sense of ratio might be involved in processing symbolic fractions.

\section{The current study}

The goal of the current study is twofold. First, we aimed to provide further evidence for the universality of the RPS by exploring to what extent individuals with a range of math skills are perceptually sensitive to non-symbolic ratio magnitudes. To this end, we used a match-to-sample task in which participants were asked to match a non-symbolic ratio (a pair of lines) presented on the left side of a screen (i.e., target) to one of two non-symbolic ratios presented on the right side (i.e., the match and distractor) (see Figure 1A). Critically, the ratio between the match and the distractor (i.e., the RoR) was systematically varied, such that is was small in some trials and large in others. This task was adapted from a preliminary study by Matthews (2015), in which an RoR effect (i.e., more accurate performance for large than small RoR) was found in both adults and 16 children from kindergarten. This suggests an early developing sensitivity to differences in nonsymbolic ratios, consistent with the RPS. In the present study, we aimed to replicate these preliminary findings by testing individuals who are proficient in fraction processing (i.e., adults) and individuals who are not (i.e., elementary-school children). We also aimed to test whether adults with dyscalculia (i.e., a learning disability that severely affects the acquisition of math skills; Price \& Ansari, 2013, Iuculano, 2016) exhibit an RoR effect in that task. Indeed, it has been argued that a main cause of dyscalculia is a deficit in processing approximate non-symbolic numerosities (Mazzocco, Feigenson, \& Halberda, 2011; Piazza et al., 2010). A major question, 


\section{RPS AND SYMBOLIC FRACTIONS}

however, is whether this deficit may extend to the RPS or if individuals with dyscalculia have a relatively intact RPS despite poor calculation skills.

A second goal of the present study was to examine to what extent the RPS remains involved when participants have to match symbolic fractions (rather than non-symbolic ratios) in this match-to-sample task. In other words, we aimed to examine whether participants remain sensitive to ratio magnitudes when those are expressed as symbols. Although it has been argued that the RPS may contribute to symbolic fraction understanding (Lewis, Matthews, Hubbard, 2016), prior studies have largely relied on magnitude comparison tasks that only require participants to access and estimate the approximate value of fractions. In contrast, a match-tosample task would involve asking participants to find which of two fractions is equivalent to a target fraction, which may encourage them to access the exact values of the fractions (though strategies based on approximation may still be involved to some extent). On the one hand, this task might trigger explicit calculation strategies that rely on components of fractions (i.e., numerator and denominator) and have little to do with the RPS. On the other hand, if symbolic fraction understanding is grounded in the RPS, participants may still intuitively access the RPS even in such match-to-sample tasks. To test between these possibilities, we adapted the match-tosample task described above and designed a condition in which the pairs of lines were replaced by symbolic fractions representing the same ratios (see Figure 1B). We reasoned that, if the RPS contributes to the understanding of symbolic fractions in this task, there should be an RoR effect similar to that obtained with non-symbolic ratios. In contrast, if the RPS does not contribute to the understanding of symbolic fractions, performance should not depend on RoR. 


\section{RPS AND SYMBOLIC FRACTIONS}

\section{Experiment 1}

\subsection{Methods \\ 2.1.1. Participants}

It has been argued that an effect size of $d=0.4$ is a good estimate of the smallest effect size of interest in experimental psychology (Brysbaert, 2019). Therefore, to be able to detect an effect of that size with $80 \%$ power in a within-subject comparison, we planned to recruit about 50 participants.

Forty-eight individuals from the University of Lyon volunteered to participate in this experiment. Data collection for the participants was carried out in two days. Six participants were excluded because of technological issues $(n=2)$ and non-compliance with the instructions $(n=4)$. The remaining forty-two participants $(M=20.7$ years, range $=18-54,31$ females $)$ were included in the analyses, for an achieved power of $72 \%$ (based on a hypothesized effect size of $d=0.4$ ). As compensation, participants received a book or chocolate. The experiment was performed in accordance with the ethical standards established by the Declaration of Helsinki.

\subsubsection{Materials}

Participants completed a match-to-sample task in which they were asked to match a ratio presented on the left of the screen to one of two ratios presented on the right of the screen (one of which was equivalent to the ratio on the left). The task was adapted from Matthews (2015). In two separate blocks, ratios were presented either as symbolic fractions or as non-symbolic ratios

\section{(Figure 1).}




\section{RPS AND SYMBOLIC FRACTIONS}

\subsubsection{Symbolic fractions}

Symbolic fractions were composed of numerators and denominators ranging from 1 to 15 and were presented in a font size of 20 point in Times New Roman. In each trial, 3 fractions (target, match, distractor) were presented simultaneously. The target was presented on the left of the screen and the match and distractor were presented on the right (see Figure 1). The ratio between the fractions presented as match and distractor (i.e., a Ratio of Ratios or RoR) was varied such that it was large in half of the trials and small in the other half. Large RoR were of 3:1 and 2:1. Small RoR were of 4:3 and 6:5. For instance, for a 4:3 RoR, a $2 / 3$ match could be paired with a 4/8 distractor. These ratios were chosen based on Matthews (2015). Specifically, they corresponded to the two largest and two smallest ratios from this preliminary study. For each RoR, forty unique fraction pairs were generated (see Appendix). Following previous studies (Meert, Gregoire, \& Noel, 2010; Schneider \& Siegler, 2010), we also generated an equal mixture of fractions where numerators of target and distractor were either the same or different. Critically, because there is often a relation between overall fraction magnitude and size of the numerator, we found the ratio between numerators of match and distractor to increase with that of the RoR. That is, small and large RoRs were associated with average ratios between numerators of match and distractor of 1.44 and 2.37 (respectively). This raises the possibility that participants might just compare the numerators of match and distractor to complete the task. To evaluate if this was the case, fraction pairs within small and large RoRs were further broken down into pairs for which the ratio between numerators of match and distractor was small (i.e., smaller than 1.44 for small RoRs and smaller than 2.37 for large RoRs) and pairs for which the ratio between numerators of match and distractor was relatively larger (i.e., larger than 1.44 for small RoRs and larger than 
2.37 for large RoRs). This allowed us to explore the effects of RoR and effects of ratio between numerators of match and distractor independently.

\subsubsection{Non-symbolic ratios}

Non-symbolic ratios were composed of pairs of line segments representing the same ratios as those used in symbolic fractions. Individual line lengths ranged between 10px to 160px approximately on an $800 \times 994 \mathrm{px}$ full screen. This corresponded to a size between $0.2 \mathrm{~cm}$ and 3.2 $\mathrm{cm}$ (visual angle 0.19 - 3.05 degrees). As for symbolic fractions, the ratio between the nonsymbolic ratios presented as match and distractor was varied, such that it was large (i.e., 3:1 and $2: 1)$ in half of the trials and small $(4: 3$ and $6: 5)$ in the other half. For each RoR, 40 non-symbolic ratio pairs were generated. Since the ratio size determined the magnitude of the line lengths, participants could not rely on individual line lengths and had to process the relationship between the two lines. For instance, for line ratios such as those shown in Figure 1, all the stimuli (target, match, and distractor) had different sizes of individual components. Further, a ratio of $2 / 4$ when matched to a ratio of 5/10 was composed of line segments of two/four and five/ten units of length respectively where a single unit length of line segment was fixed in size. Because of this, the individual components of the line ratios differed in size but the relationship between the ratios stayed the same. In the following analyses, we consider the "numerator" of each pair of line to be the shortest line of the pair.

\subsubsection{Procedure}

During the entire experiment, participants were seated approximately $60 \mathrm{~cm}$ from a 14 '” screen. All instructions were presented on the laptop screen in French. Participants could ask 
questions about the task after reading the instructions and receive additional instructions if they needed to. Participants were told that they would see in each trial 3 ratios (one on the left side of the screen and 2 on the right side) presented in either symbolic or non-symbolic format. For both notations, they were instructed to match the ratio on the left (i.e., the target) to the equivalent ratio on the right (i.e., the match) as accurately and quickly as possible. They could do so by pressing the button ' $\mathrm{H}$ ' if the upper stimulus was equivalent to the target and the button ' $\mathrm{B}$ ' if the lower stimulus was equivalent to the target (on a QWERTY keyboard). The 3 ratios were presented for a duration of 3,000 ms. Participants could respond from the onset of stimuli until the timeout at 6,000 ms.

Participants completed 1 block of non-symbolic ratios and 1 block of symbolic fractions. Each block included 4 practice trials (with feedback) that were followed by 40 experimental trials. Each block was made of 20 trials with a large RoR (i.e., 10 trials with a RoR of 3:1 and 10 trials with a RoR of 2:1) and 20 trials with a small RoR (i.e., 10 trials with a RoR of 4:3 and 10 trials with a RoR of 6:5). These trials were selected randomly from the triplets of fractions generated for each RoR. The practice trials were constructed of easier RoR (4:1) and participants had to reach $100 \%$ accuracy to be presented with experimental trials. Almost all participants were able to complete the practice trials with $100 \%$ accuracy. Those who did not were given additional instructions and were successful in completing the practice trials. Block order was counterbalanced and trial order was randomized within blocks. Side of the presentation of the match was counterbalanced with half of the match ratios on the top and the other half at the bottom. The experiment was designed using JavaScript and HTML. The entire experiment lasted between 10 and $15 \mathrm{~min}$. 
RPS AND SYMBOLIC FRACTIONS

\subsection{Results}

Proportion correct was analysed in a repeated-measures ANOVA with the within-subject factors RoR (Large, Small), Notation (Line, Fraction), Ratio between numerators of match and distractor (Large, Small), and Numerator of the distractor (Identical to the target, Different from the target). All analyses were carried out in Jamovi (www.jamovi.org/). Main effects and interactions relevant to our hypotheses are presented below.

First, this ANOVA revealed a main effect of Notation $\left(F(1,41)=16.36, p<0.001, \eta^{2}{ }_{p}=\right.$ $0.285)$ and a main effect $\operatorname{RoR}\left(F(1,41)=22.96, p<0.001, \eta_{\mathrm{p}}^{2}=0.359\right)$, which were qualified by an interaction between Notation and $\operatorname{RoR}\left(F(1,41)=49.79, p<0.001, \eta^{2} \mathrm{p}=0.548\right)$. Bonferroni corrected t-tests revealed that participants were more accurate for large than small RoR when choosing between non-symbolic ratios $(p<0.001)$. However, there was no accuracy difference between large and small RoR when choosing between symbolic fractions $(p=0.701)$. This interaction between Notation and RoR was neither affected by Numerator of the distractor $(F$ $\left.(1,41)=0.45, p=0.505, \eta^{2} \mathrm{p}=0.011\right)$ nor by Ratio between numerators of match and distractor $\left(F(1,41)=0.33, p=0.567, \eta_{p}^{2}=0.008\right)$. Thus, RoR differently affected performance on nonsymbolic and symbolic stimuli across all different trial types (see Figure 2).

Second, there was a significant interaction between Notation and Numerator of the distractor $\left(F(1,41)=32.62, p<0.001, \eta_{\mathrm{p}}^{2}=0.443\right)$, revealing that participants were most accurate when the numerator of the distractor was different from the target for non-symbolic ratios $(p<0.001)$ whereas they were most accurate when numerators were identical across target and distractor for symbolic fractions $(p<0.001)$.

Third, the ANOVA revealed a main effect of Ratio between numerators of match and distractor $\left(F(1,41)=5.53, p=0.024, \eta_{p}^{2}=0.119\right)$, which was qualified by an interaction with 
Notation $\left(F(1,41)=40.26, p<0.001, \eta^{2}{ }_{p}=0.495\right)$. For non-symbolic ratios, participants were more accurate when the ratio between numerators of match and distractor was large than small $(p$ $<0.001)$. For symbolic fractions, however, performance was not affected by the ratio between numerators of match and distractor $(p=0.378)$.

Because frequentist statistics do not provide evidence for a null hypothesis, we turned to Bayesian statistics (Morey et al., 2016; Lee and Wagenmakers, 2013) to estimate the strength of evidence (i.e., the Bayes factor, BF) for a lack of difference between large and small RoR (H0) versus a higher accuracy for large than small RoR (H1) in both symbolic and non-symbolic trials. Following Jeffreys (1961), a $\mathrm{BF}<3$ was considered anecdotal evidence, a $3<\mathrm{BF}<10$ was considered substantial evidence, a $10<\mathrm{BF}<30$ was considered strong evidence, a $30<\mathrm{BF}<100$ was considered very strong evidence and a BF $>100$ was considered extreme evidence that our data are more likely under the alternate than the null hypothesis (i.e., $\mathrm{BF}_{+0}$ ) or under the null hypothesis than the alternate hypothesis (i.e., $\mathrm{BF}_{0^{+}}$). Bayesian paired t-tests with default priors (0.707) revealed strong evidence for $\mathrm{H} 0$ versus $\mathrm{H} 1$ in the case of symbolic fractions $\left(\mathrm{BF}_{0^{+}}=\right.$ 14.4). In contrast, there was extreme evidence for $\mathrm{H} 1$ versus $\mathrm{H} 0$ in the case of non-symbolic ratios $\left(\mathrm{BF}_{+0}>100\right)$. Therefore, while there was evidence that large RoR were more accurate than small RoR for non-symbolic ratios, there was also evidence that it was not the case for symbolic fractions.

\subsection{Discussion}

In this first experiment, we explored the extent to which typical adults are sensitive to ratio magnitudes when they match either non-symbolic ratios or symbolic fractions. The findings demonstrate a clear RoR effect for non-symbolic line ratios, with higher accuracy for large RoR 
than for small RoR. These findings replicate the preliminary results described in Matthews (2015). They also suggest that adults are perceptually sensitive to ratio magnitudes, even when they have to match (and not only compare, as in magnitude comparison tasks) non-symbolic stimuli. This supports the claim that adults possess an RPS that may allow them to access a perceptual sense of ratios, akin to the ANS for whole numerosities (Dehaene, 1997; Feigenson, Dehaene, \& Spelke, 2004; Gilmore, Attridge, De Smedt, \& Inglis, 2014). Interestingly, our study indicates that some componential strategies may also be involved in this task. For example, in addition to the RoR effect, accuracy depended on whether numerators of target and distractor (i.e., the shortest line of each pair) were identical or different. It also depended on the ratio between numerators of match and distractor. This suggests that participants may have attended to components of each pair of lines in addition to their overall ratio. This is broadly consistent with previous findings that have shown that participants may rely on both holistic and componential strategies when comparing fractions (though these studies mainly focused on symbolic fractions) (Bonato et al., 2007; Faulkenberry \& Pierce, 2011; Meert et al., 2010, 2012; Obersteiner et al., 2013; Schneider \& Siegler, 2010).

A novel aspect of our study is that we asked participants to match symbolic fractions in addition to non-symbolic ratios. In sharp contrast to the results obtained with non-symbolic ratios, our results revealed an absence of RoR effect for symbolic fractions. This suggests that participants do not access a representation of fractional magnitudes when they have to match symbolic fractions in this task. Rather, they are likely to use componential strategies, focusing on the numerators and denominators separately. This is suggested by the fact that participants' accuracy depended on whether the numerator of the target was identical or different from the numerator of the distractor. Clearly, focusing on the numerator is an efficient strategy when the numerator of the target is the same as the distractor because it indicates that these fractions are 


\section{RPS AND SYMBOLIC FRACTIONS}

not equivalent. However, the effectiveness of such a componential strategy is less obvious when numerators differ between target and distractor. Yet, our results clearly indicate that performance does not depend on RoR, suggesting that participants do not rely on fractional magnitudes to complete the task.

Other strategies that participants are likely to rely on in Experiment 1 may involve calculating (e.g., reducing a fraction to its simplest form) and their mastery may emerge after years of practice in school. Therefore, reliance on these strategies might depend on degree of familiarity with fractions as well as proficiency with arithmetic. Such strategies may also tax working memory and therefore depend on working memory resources of participants. In other words, it is possible that participants with limited practice with manipulating symbolic fractions, limited arithmetic fluency, or limited working memory resources may rely on an intuitive sense of fractional magnitudes to a greater extent than competent adults. To test this hypothesis, we presented the same task to children who are beginning to learn about symbolic fractions in $4^{\text {th }}$ and $5^{\text {th }}$ grade. These children should, therefore, have more limited experience with the strategies used to solve fraction problems than adults. They should also have lower arithmetic fluency and working memory ability than adults (Cowan, 2016; De Brauwer, Verguts, \& Fias, 2006; Imbo \& Vandierendonck, 2008). None of these factors may influence the RPS because it is thought to be an evolutionarily old system that requires few cognitive resources and is early developing. However, these factors may affect the extent to which children use strategies in the symbolic version of the task. Therefore, we predicted that these children should rely (at least to some extent) on the RPS to match symbolic fractions as well as non-symbolic ratios (i.e., they should exhibit a sensitivity to ratio magnitudes when matching non-symbolic ratios and symbolic fractions). 


\section{RPS AND SYMBOLIC FRACTIONS}

\section{Experiment 2}

\subsection{Methods \\ 3.1.1. Participants}

Children are introduced to symbolic fractions during $4^{\text {th }}$ grade in France. Thus, we recruited $404^{\text {th }}$ and $5^{\text {th }}$ graders from a private school in Lyon, France. The difference between large and small RoR for non-symbolic stimuli in Experiment 1 was very large (Cohen's d = 1.43). Therefore, this sample size, which would yield more than $99 \%$ power to detect an effect size of $d=0.80$ in a within-subject comparison, was more than adequately powered to detect an effect of that size. Two children were excluded because of non-compliance with instructions. The remaining thirty-eight children $(M=9.92$ years, range $9-11,26$ females $)$, twenty-one $4^{\text {th }}$ graders and seventeen $5^{\text {th }}$ graders were included in the study. Data collection was done during one school day. The experiment was performed in accordance with the ethical standards established by the Declaration of Helsinki.

\subsubsection{Materials and Procedure}

Materials and procedures remained the same as in Experiment 1. However, children were given oral rather than written instructions about the task and were tested in groups of four. 
RPS AND SYMBOLIC FRACTIONS

\subsection{Results}

Proportion correct was analysed in a repeated-measures ANOVA with the factors RoR (Large, Small), Notation (Line, Fraction), Ratio between numerators of match and distractor (Large, Small), and Numerator of the distractor (Identical to the target, Different from the target).

First, the ANOVA revealed a main effect of $\operatorname{RoR}\left(F(1,37)=14.74, p<0.001, \eta_{p}^{2}=\right.$ 0.285), which was qualified by an interaction between RoR and Notation $(F(1,37)=39.76, p<$ $\left.0.001, \eta_{\mathrm{p}}^{2}=0.518\right)$. Bonferrori-corrected post hoc tests showed that children were more accurate for large than small RoR for non-symbolic ratios $(p<0.001)$. In contrast, there was no difference in accuracy between large and small RoR for symbolic fractions $(p=1.000)$. The interaction between Notation and RoR was neither affected by Numerator of the distractor $(F(1,37)=0.56$, $\left.p=0.460, \eta_{\mathrm{p}}^{2}=0.015\right)$ nor by Ratio between numerators of match and distractor $(F(1,37)=$ 0.55, $\left.p=0.462, \eta_{\mathrm{p}}^{2}=0.015\right)$. Thus, much like in typical adults, RoR differently affected performance on non-symbolic and symbolic stimuli across all different trial types in children (see Figure 3).

Second, the ANOVA revealed a main effect of Ratio between numerators of match and distractor $\left(F(1,37)=11.42, p=0.002, \eta^{2}{ }_{p}=0.236\right)$. Although the interaction with Notation did not reach significance $\left(F(1,37)=1.51, p=0.227, \eta_{p}^{2}=0.039\right)$, follow-up Bonferroni tests indicated that the main effect of Ratio between numerators of match and distractor was driven by non-symbolic stimuli. For non-symbolic ratios, children were more accurate when the ratio between numerators of match and distractor was large than small $(p=0.011)$. For symbolic fractions, performance was not affected by the ratio between numerators of match and distractor $(p=0.892)$. 
Bayesian paired t-tests with default priors (0.707) revealed extreme evidence for H1 (i.e., large RoR is associated with higher proportion correct than small RoR) versus H0 (i.e., no difference between large and small RoR) when children had to choose between non-symbolic ratios $\left(\mathrm{BF}_{+0}>100\right)$. There was, however, moderate evidence for $\mathrm{H} 0$ versus $\mathrm{H} 1$ when children had to choose between symbolic fractions $\left(\mathrm{BF}_{0+}=6.92\right)$. Therefore, much like adults in Experiment 1, there was evidence with children that large RoR were more accurate than small RoR for nonsymbolic ratios. However, there was also evidence that it was not the case for symbolic fractions.

\subsection{Discussion}

In Experiment 2, we presented the symbolic and non-symbolic versions of the match-tosample task to children in $4^{\text {th }}$ and $5^{\text {th }}$ grade. Because children are introduced to symbolic fractions in $4^{\text {th }}$ grade in France, we reasoned that these participants may have less experience with manipulating symbolic fractions than adults and lack practice with different strategies. Accordingly, they may rely to a lesser extent on these strategies when matching symbolic fractions (and might instead rely on a more intuitive sense of ratio magnitudes). Consistent with the fact that these participants are less proficient with symbolic fractions than adults, children matched symbolic fractions with a relatively low accuracy of about $54.6 \%$ (as opposed to $73.8 \%$ on average for adults in Experiment 1). Accuracy was also comparable in trials in which the numerator of the target fraction was similar to that of the distractor fraction $(54.1 \%)$ and in trials in which the numerator of the target fraction was different from that of the distractor fraction $(55.2 \%)$. This suggests that children struggled to perform this symbolic version of the task and focus less systematically on numerators than adults did in Experiment 2 (similar to Zhang, Fang, Gabriel, \& Szücs, 2014). Yet, the pattern of results is largely similar to Experiment 1. 
First, children exhibited RoR effects when matching non-symbolic ratios, indicating that children around the age of 10 are perceptually sensitive to ratio magnitudes. This is in line with several prior studies also showing sensitivity to non-symbolic ratio magnitudes in children. For example, in the preliminary study by Matthews (2005), sixteen pre-schoolers showed RoR effects when matching non-symbolic ratios. In another study, six-month-old infants show similar discrimination acuity for non-symbolic ratios as they do for non-symbolic numerosities in magnitude discrimination tasks (McCrink \& Wynn, 2007). Finally, studies on non-symbolic proportional reasoning tasks employing continuous quantities (in which participants have to focus on the relationship between the quantities because individual units cannot be counted) have shown that children as young as 6 years old are successful in processing proportional information across a wide range of non-symbolic formats (Boyer, Levine, \& Huttenlocher, 2008; Hurst \& Cordes, 2018; Jeong, Levine, \& Huttenlocher, 2007; Meert, Grégoire, Seron, \& Noël, 2013). Therefore, together with these studies, our data are consistent with the idea that the RPS is relatively early developing.

Second, despite a level of accuracy relatively similar between the non-symbolic ratios and the symbolic fractions (49.4\% versus $54.6 \%$ ), children's accuracy in the symbolic task was not dependent upon the RoR. Therefore, contrary to our prediction, even children with a lack of extensive experience with componential and other strategies do not appear to access representations of magnitude when matching symbolic fractions. Of course, children are beginning to learn about fractions in $4^{\text {th }}$ grade, as indicated by relatively high error rates. It is thus possible that they were not exposed to enough instruction on rational numbers to be able to access the underlying ratio magnitudes. To test this possibility, we turned to adults with dyscalculia. 
Dyscalculia is a specific learning disability affecting the acquisition of numerical skills, despite normal intelligence and educational opportunities. Research has shown that individuals with developmental dyscalculia possess an impaired ANS as well as relatively poor working memory ability (Geary, 2011; Iuculano, 2016; Mazzocco et al., 2011; Piazza et al., 2010). This, in turn, is thought to significantly affect arithmetic learning. Clearly, adults with dyscalculia have been exposed to extensive instruction on rational numbers in school. Yet, their impaired calculation skills may prevent them from effectively using componential strategies when matching symbolic fractions. Although this may translate into greater use of the RPS, it is also possible that ANS impairments in individuals with dyscalculia may extend to the RPS. Therefore, Experiment 3 investigated the sensitivity to ratio magnitudes of adults with dyscalculia who were asked to match both non-symbolic ratios and symbolic fractions.

\section{Experiment 3}

\subsection{Methods \\ 4.1.1. Participants}

Seventeen adult participants were recruited using advertisements in social media, the university "mission-handicap", and by word of mouth. Thirteen participants $(M=23.3$ years, range 19-27, 12 females) met the inclusion criteria based on clinical diagnosis and standardized tests (described below), yielding $80 \%$ power to detect an effect size of $d=0.85$ in a withinsubject comparison. Because the effect sizes associated with the difference in accuracy between large and small RoR in Experiments 1 and 2 were larger than $\mathrm{d}=0.85$ (i.e., $\mathrm{d}=1.43$ and $\mathrm{d}=$ 


\section{RPS AND SYMBOLIC FRACTIONS}

1.08), we considered this sample size to be adequate. The experiment was performed in accordance with the ethical standards established by the Declaration of Helsinki.

\subsubsection{Standardized Tests}

Adults were administered various tests to assess cognitive, numerical, and reading skills. Using the WAIS-IV (Wechsler, 2008), verbal IQ was estimated using the verbal reasoning subtest while spatial IQ was estimated using the matrix reasoning subtest. Math skills were assessed using 2 subtests of the Woodcock-Johnson Test of Achievement (WJ-III) (Woodcock et al., 2001): Math Fluency and Applied Problems. The Math Fluency sub-test is a timed test in which the participants have to solve as many single-digit additions, subtraction, multiplication, and division problems as they can within 3 min. The Applied Problems subtest is un-timed and measures the ability to analyse basic numerical concepts and oral word problems. This test stops after 6 consecutive errors or when the last item is reached. Reading fluency was assessed with the Alouette-R test (Lefavrais, 1967), which is frequently used to test reading skills in Frenchspeaking countries. This test requires participants to read a 265 -word text aloud in 3 minutes and measures the number of words read, the time required, and the number of pronunciation errors to evaluate the reading speed and efficiency, respectively. Because the test is standardized for children and adolescents but not for adults, we used the guidelines of Cavalli et al. (2018) to identify cut-off criteria for dyslexia (i.e., reading speed score above 8.7 and/or reading efficiency score above 402.26)

Psychometric measures are shown in Table 1. To be classified as having dyscalculia, participants had to have been diagnosed by a clinical specialist, complained of mathematics 
difficulties since school, and perform at or below the $25^{\text {th }}$ percentile on at least one of the math sub-tests of fluency and applied problems (Jordan et al., 2003). Additionally, all 13 participants had to have a normal verbal IQ, as reflected by a score above the $30^{\text {th }}$ percentile (i.e., 92) on the verbal reasoning subtest of the WAIS-IV. All but three participants had reading scores above the cut-off criterion for dyslexia (Cavalli et al., 2018).

\section{--- Insert Table 1 about here ---}

\subsubsection{Materials and Procedures}

Materials and procedures remained the same as in Experiment 1 with one exception. Because the sample size was smaller than in Experiment 1, we presented participants in Experiment 3 with twice more trials to improve individual estimates of performance. That is, after completing 4 practice trials in each block, participants evaluated 80 experimental trials. Therefore, there was a total of 160 trials (i.e., 80 non-symbolic ratios and 80 symbolic fractions). The whole experiment lasted about 20 minutes.

\subsection{Results}

As for Experiment 1 and Experiment 2, proportion correct was analysed in a repeatedmeasures ANOVA with RoR (Large, Small), Notation (Line, Fraction), Ratio between numerators of match and distractor (Large, Small), and Numerator of the distractor (Identical to the target, Different from the target) as within-subject factors. 
First, this ANOVA revealed an interaction between Notation and $\operatorname{RoR}(F(1,12)=35.04$, $\left.p<0.001, \eta_{\mathrm{p}}^{2}=0.745\right)$. Bonferroni corrected t-tests revealed that participants were more accurate for large than small RoR when choosing between non-symbolic ratios $(\mathrm{p}<0.001)$. However, there was no accuracy difference between large and small RoR when choosing between symbolic fractions $(\mathrm{p}=0.106)$. This interaction between Notation and RoR was neither affected by Numerator of the distractor $\left(F(1,12)=0.36, p=0.557, \eta^{2}{ }_{p}=0.029\right)$ nor by Ratio between numerators of match and distractor $\left(F(1,12)=1.47, p=0.248, \eta_{\mathrm{p}}^{2}=0.109\right)$. Thus, as in Experiment 1 and 2, RoR differently affected performance on non-symbolic and symbolic stimuli across all different trial types in adults with dyscalculia (see Figure 4).

Second, there was a significant interaction between Notation and Numerator of the distractor $\left(F(1,12)=20.09, p<0.001, \eta_{p}^{2}=0.626\right)$, revealing that participants were most accurate when the numerator of the distractor was different from the target for non-symbolic ratios $(p=0.017)$ whereas this factor was no affecting accuracy for symbolic fractions $(p=$ 1.000).

Third, the ANOVA revealed a main effect of Ratio between numerators of match and distractor $\left(F(1,12)=22.17, p<0.001, \eta^{2}{ }_{p}=0.649\right)$, which was qualified by an interaction with Notation $\left(F(1,12)=11.47, p=0.005, \eta^{2}{ }_{p}=0.489\right)$. For non-symbolic ratios, participants were more accurate when the ratio between numerators of match and distractor was large than small $(p$ $<0.001)$. For symbolic fractions, however, performance was not affected by the ratio between numerators of match and distractor $(p=0.522)$.

Bayesian paired t-tests with default priors (0.707) revealed extreme evidence for H1 (i.e., large RoR is associated with higher proportion correct than small RoR) versus H0 (i.e., no difference between large and small RoR) when participants had to choose between non-symbolic 
ratios $\left(\mathrm{BF}_{+0}>100\right)$. There was moderate evidence for $\mathrm{H} 0$ versus $\mathrm{H} 1$ when participants had to choose between symbolic fractions $\left(\mathrm{BF}_{0^{+}}=10.89\right)$. Thus, adults with dyscalculia displayed higher accuracy for large than small RoR for non-symbolic ratios, but not for symbolic fractions.

\subsection{Discussion}

Results of Experiment 3 show that adults with dyscalculia exhibit a pattern of responses very similar to that of children in Experiment 2. First, overall accuracy was low on both nonsymbolic ratios (54\%) and symbolic fractions (62.6\%), suggesting that participants completed the tasks with some difficulty. Second, despite this relatively low accuracy, there was a clear RoR effect with non-symbolic ratios. This suggests that adults with dyscalculia have a perceptual sensitivity to ratio magnitudes, much like normal adults in Experiment 1 and children in Experiment 2. This is noteworthy because it presents evidence that adults with dyscalculia may have access to a relatively intact RPS. Interestingly, prior research on number acuity has revealed an impaired ANS in individuals with dyscalculia (Mazzocco et al., 2011; Piazza et al., 2010 for a contrasting view see Iuculano, Tang, Hall, \& Butterworth, 2008). To our knowledge, the present study is the first to examine the intuitive, perceptual ability to process non-symbolic ratios in this population. Our findings raise the possibility that the RPS may be relatively independent from the ANS and spared in dyscalculia. Note, however, that these results come from a relatively small sample size (despite moderate to large effect size, $\eta_{\mathrm{p}}^{2}=0.346$ ) and that future studies would need to confirm this claim by gathering concomitant measures of both ANS and RPS in individuals with dyscalculia. 


\section{RPS AND SYMBOLIC FRACTIONS}

Third, we expected to observe a RoR effect for symbolic fractions, due to less automatized calculative abilities and lower working memory capacity in adults with dyscalculia. However, performance on symbolic fractions was not affected by RoR, indicating that adults with dyscalculia do not access a representation of ratio magnitudes when they match symbolic fractions.

Overall, results of Experiment 3 are similar to the results of Experiment 1 and Experiment 2 in that participants do not naturally appear to use the RPS to match symbolic fractions in this task, independently of their level of math skills. This, however, may not mean that they cannot use the RPS when processing symbolic fractions. For example, accessing the RPS may be necessary if participants are forced to estimate the ratio that underlie a symbolic fraction. To explore this hypothesis, we designed a mixed-format version of the match-to-sample task in which another sample of adults were asked to choose which of two symbolic fractions corresponds to a non-symbolic ratio (see Figure 5). In such a task, participants are forced to estimate (rather than precisely determine) which symbolic fraction is the best match for the nonsymbolic ratio. We expected that such a mixed-format task would require participants to access fractional magnitudes in a way that previous symbolic versions of the task did not.

\section{Experiment 4}

\subsection{Participants}

Thirty-three participants $(M=20.5$ years, range 18-25, 25 females $)$ from the University of Lyon 2 volunteered to participate in the study. All of them were included in the analyses. This yields $80 \%$ power to detect an effect size of $d=0.50$ (which would be much smaller than the 
effect reported for the difference between large and small RoR in non-symbolic stimuli in the previous experiments). The experiment was performed in accordance with the ethical standards established by the Declaration of Helsinki.

\subsubsection{Materials and Procedures}

The procedure remained the same as in the experiments above. However, stimuli for the match-to-sample task were modified. For this task, non-symbolic ratios were presented on the left side of the screen and symbolic fractions were presented on the right side of the screen (see Figure 5). Participants were asked to match the non-symbolic ratio on the left with the correct symbolic fraction on the right. Participants completed one block with 4 practice trials (with feedback) followed by 80 experimental trials (consisting of 20 trials per RoR). The entire experiment lasted for about 10 minutes.

\subsection{Results}

Proportion correct was analysed in a repeated-measures ANOVA with the factor RoR (Large, Small). The analysis revealed a main effect of $\operatorname{RoR}\left(F(1,32)=63.9, p<0.001, \eta^{2}=\right.$ 0.666), indicating that participants were more accurate for large RoR than small RoR (see Figure 6).

In line with the results above, a Bayesian paired t-test with default priors $(0.707)$ revealed extreme evidence for H1 (i.e., large RoR are associated with higher proportion correct than small RoR) versus H0 (i.e., no difference between large and small RoR) across all trials of the 


\section{RPS AND SYMBOLIC FRACTIONS}

experiment $\left(\mathrm{BF}_{+0}>100\right)$. Therefore, there was evidence for a difference in accuracy between large and small RoR.

\subsection{Discussion}

Results of Experiment 4 clearly show an RoR effect in this mixed-format version of the match-to-sample task, indicating that adult participants do access fractional magnitudes to complete this task. This demonstrates that the lack of RoR effect in symbolic fractions in Experiments 1 to 3 may not necessarily reflect an inability to use the RPS to process symbolic fractions. Rather, it suggests that participants do not naturally access this non-symbolic representation when they are asked to match symbolic fractions and are most likely reverting to componential strategies. Yet, it is interesting to note that overall accuracy in Experiment 4 is reliably above chance $(66 \%, \mathrm{t}(32)=7.48, p<0.001, \mathrm{~d}=1.30)$, suggesting that strategies relying on an intuitive understanding of fractional magnitudes may be relatively efficient. The results of the four experiments are discussed in detail below.

\section{General Discussion}

In the above experiments, we aimed to test the hypothesis that participants are sensitive to ratio magnitudes when they match non-symbolic ratios as well as symbolic fractions, thereby providing further evidence for the existence of the RPS and its potential role in symbolic fraction processing. Using a version of a match-to-sample task developed by Matthew (2015), we asked participants to choose which of two non-symbolic ratios or symbolic fractions corresponded to a given non-symbolic ratio or fraction. Our results indicate that adults have a perceptual sensitivity 
to ratios when these are presented in a non-symbolic format. This sensitivity does not appear to be affected by the level of math skill because it was also largely present in individuals with lower math abilities (i.e., children in $4^{\text {th }}$ and $5^{\text {th }}$ grade and adults with dyscalculia). Furthermore, this sensitivity was observed when symbolic fractions had to be matched with a non-symbolic ratio, suggesting that the RPS may contribute to symbolic fraction processing in some situations. However, performance did not depend on the RoR when symbolic fractions had to be matched with other symbolic fractions.

\section{A perceptual route to process the approximate magnitude of fractions}

Over our first 3 experiments, the results clearly indicate that participants' performance is affected by the RoR when they match non-symbolic ratios. Therefore, our results add to the growing evidence that the human cognitive architecture provides a perceptual route to process approximate magnitudes of fractions when represented by non-symbolic ratios (Fazio, Bailey, Thompson, \& Siegler, 2014; Matthews \& Chesney, 2015; Matthews \& Lewis, 2017). For instance, Matthew (2015) noticed that pre-schoolers may already possess some perceptual sensitivity towards non-symbolic ratios and that the accuracy of some high achieving preschoolers might match adult levels (Matthews, 2015; Matthews \& Ziols, 2019). Furthermore, this sensitivity has been shown to be present across species, age groups, and even in societies that lack formal mathematic education (Jacob et al., 2012; Jeong et al., 2007; Kalra et al., 2020; McCrink et al., 2013; McCrink \& Wynn, 2007; Meert et al., 2012, 2013; Sophian, 2000). Thus, this evidence points towards the RPS being an early developing, evolutionarily ancient system (Lewis, Matthews, \& Hubbard, 2016). 
We also observed that performance of $4^{\text {th }}$ and $5^{\text {th }}$ graders on the non-symbolic ratio comparison task in Experiment 2 was much lower than that of adults in Experiment 1 (contrary to the preliminary study by Matthews, 2015). This might be due to the fact that the RPS might still be developing in children and/or that formal mathematical experience might influence RPS acuity. This would be relatively consistent with research on the ANS, which shows that formal mathematical experience typically improves ANS acuity (Halberda et al., 2008; Pica, 2004). Similarly, even though the RPS might be an evolutionarily ancient system present early on in developmental years, its acuity might be dependent on both the quantity and quality of mathematical experience and the age.

Finally, we found clear RoR effects for non-symbolic line ratios in adults with dyscalculia. Dyscalculia is a complex, multifaceted neurodevelopmental disorder that hinders acquisition of mathematical concepts despite normal intelligence (Iuculano, 2016). Much of the research on dyscalculia has focused on whether and how the disability affects the processing of whole number magnitudes. Some researchers have identified ANS deficits to be a potential source of dyscalculia (Butterworth, 2005, 2010; Mazzocco et al., 2011; for a contrasting view, see De Smedt \& Gilmore, 2011; Iuculano et al., 2008, Rousselle \& Noel, 2007). To our knowledge no research on perceptual sensitivity of relational magnitudes in adults with dyscalculia exists. Our results in adults with dyscalculia highlight two important points. First, the fact that performance of adults with dyscalculia depends on RoR in our non-symbolic task suggests that these individuals possess the perceptual ability to process ratios. This naturally suggests a spare RPS in adults with dyscalculia. Second, performance of adults with dyscalculia in this task was much lower than that of typical adults, which could be attributed either to the quality of mathematical experience or a slower development of the RPS system. Nonetheless, our findings raise the intriguing possibility that even though adults with dyscalculia may experience 
difficulties with whole number concepts, they might still be able to leverage the RPS to learn fractions. One can speculate that this ability to process relational magnitudes between numbers might even be utilized to teach whole number concepts (Lewis, Matthews, Hubbard, 2016). Future studies on larger sample size and across different age groups will further help develop a comprehensive picture of the development of the RPS in individuals with dyscalculia. This knowledge might aid in current efforts to develop identification criteria and screeners for dyscalculia in children (Rodrigues \& Jordan, 2019) and adults.

\section{A limited role for the RPS in symbolic fraction processing}

Results of Experiment 4 suggests RPS involvement in symbolic fraction processing. Specifically, we found that typical adults exhibit the signature RoR effect when they have to choose between two symbolic fractions to match a non-symbolic ratio. Our results are in line with a prior study done on cross-format comparisons revealing distance effects across different formats (dot arrays, circle areas, symbolic fractions) indicating magnitude abstraction (Matthews \& Chesney, 2015). It is noteworthy to mention that several other studies on fraction magnitude comparison tasks have also demonstrated a link between perceptual sensitivity to non-symbolic ratios and symbolic fraction processing (Matthews, Chesney, \& McNeil, 2014). Furthermore, it has been argued that the cross-format comparison tasks are key to understanding a shared system for non-symbolic and symbolic magnitudes as they rely on the abstract nature of magnitude between the different notations (Matthews \& Chesney, 2015).

However, we did not find any RoR effect when participants had to choose which of two symbolic fractions matched another symbolic fraction. This was true for adults (Experiment 1), children from $4^{\text {th }}$ and $5^{\text {th }}$ grade (Experiment 2), and adults with dyscalculia (Experiment 3 ). Thus, participants do not appear to access ratio magnitudes when performing this matching task. On the 


\section{RPS AND SYMBOLIC FRACTIONS}

one hand, these findings are consistent with a body of literature showing that accessing the holistic magnitude of fractions is challenging for typical adults (Kallai \& Tzelgov, 2009, 2012; Stafylidou \& Vosniadou, 2004). On the other hand, they somewhat conflict with prior studies that suggest that the RPS contribute to symbolic fraction processing. For example, in contrast to our findings, studies have found that performance on fraction comparison tasks is influenced by linear or RoR distance (Kalra et al., 2020; Matthews \& Chesney, 2015; Schneider \& Siegler, 2010).

Interestingly, this difference between our results and those prior findings does not seem to stem from a clear difference in timing of response. For example, average response time was of 2,284 ms for adults in Experiment 1, which is only slightly higher than the average response time of 1,995 ms found in the fraction comparison task designed by (Matthews \& Chesney, 2015) (consider also that 3 fractions are displayed simultaneously in a match-to-sample task, compared to only 2 fractions in a comparison task). It is also unlikely that our participants may have chosen not to rely on fractional magnitude because they used another analogical strategy involving a comparison of the size of components of fractions. Indeed, in contrast to what was found for nonsymbolic ratios (see below), the ratio between numerators of match and distractor did not influence performance on symbolic fractions. Thus, differences between our findings and that of previous studies are more likely due to differences in the processes involved in magnitude comparison versus match-to-sample tasks. In magnitude comparison tasks, participants are asked to compare the relative sizes of two fractions (e.g., assess which fraction is larger). This task can arguably be done without accessing the exact value of each fraction. Thus, magnitude comparison tasks are likely to encourage participants to simply estimate approximate magnitudes (which might promote strategies relying on analogical processing). In our match-to-sample task, however, we asked participants to find which of two fractions corresponded to a target fraction. 
Although we acknowledge that it is possible to complete this task by approximating ratios, we speculate that such an equivalence task more likely prompts participants to access the exact rather than the approximate value of fractions. Therefore, although children, typical adults, and adults with dyscalculia may rely on an intuitive sense of ratios to process non-symbolic ratio magnitudes, our findings raise the possibility that this sense may only drive performance in tasks that encourage subjects to approximate ratios.

\section{The role of componential strategies in symbolic fraction and non-symbolic ratio processing}

Overall, our study clearly highlights an influence of components of fractions in typical adults. Interestingly, this influence was not limited to symbolic fractions but was also observed in non-symbolic line ratios. For example, in addition to RoR, accuracy in the non-symbolic task depended upon (i) whether the numerator (i.e., smallest of the lines) of the target was the same as the numerator of the distractor and (ii) whether the ratio between the numerators of the match and distractor was small or large. For symbolic fractions, accuracy was also higher when numerators were identical between match and distractor, indicating that participants might have used common components to process magnitudes. This influence of components on fraction processing is consistent with previous reports. For instance, experts have been shown to make use of componential strategies when they are efficient to solve fraction problems (Obersteiner et al., 2013). Drawing on concepts from the Dynamic Strategy Choice account (Alibali \& Sidney, 2015), we argue that the specific task feature of common numerator might have led participants to utilize a simple and effective "compare the denominator" strategy. It is also possible that the use of such a strategy with symbolic fractions might have influenced the adults to utilize a similar strategy for non-symbolic formats (in addition to the RPS). Note that it is also possible that the use of trials in which the numerator of the target was identical to that of the distractor might have 


\section{RPS AND SYMBOLIC FRACTIONS}

also encouraged participants to use componential strategies, even on other trials in which the numerator of the target was different than that of the distractor. Although this equal mixture of trials was chosen to present participants with a variety of fractions, future studies might investigate to what extent the use of componential strategies may depend on different types of fractions on such match-to-sample tasks.

This ability to utilize and form new strategies to solve problems may also develop with age and mathematical experience, consistent with the fact that the identity of the numerator (i.e., same or different across target and distractor) influenced performance in adults (Experiment 1) but not in children (Experiment 2). These results are similar to a study done by Zhang \& Colleagues, 2014, whereby based on accuracy rates the authors noted that adults are better equipped to adopt componential processing strategies than children. Finally, an important question regarding the role of formal education in shaping perceptual abilities was raised by Lewis, Matthews, \& Hubbard, 2015 in their chapter. We believe our results might have shed some light on this phenomenon. The finding that processing non-symbolic line ratios may rely on componential strategies might suggest that formal education and years of experience could be responsible for the transfer of these efficient strategies from symbolic fractions to non-symbolic line ratios thus, playing a role in shaping perceptual abilities.

\section{Symbolic fraction knowledge in adults with dyscalculia}

A couple of prior studies have investigated fraction knowledge in children with dyscalculia. These studies point to the difficulties encountered by children with dyscalculia when processing symbolic fractions. For example, it has been shown that children with dyscalculia fail to order fractions, estimate the sum of two fractions, and identify equivalent fractions (Mazzocco \& Devlin, 2008; Hansen, Jordan, \& Rodrigues, 2015). In a study by Siegler \& Pyke, 2013 and 
Bailey et al., 2015, understanding magnitudes of fractions and performing arithmetic computations were shown to be challenging for children with dyscalculia. Another study showed that children with dyscalculia also persist to view visual models of fractions as concrete representations until Grade 8, which misguides them on Arabic fraction representations (Mazzocco, Myers, Lewis, Hanich, \& Murphy, 2013). To our knowledge no studies to date have examined the difficulties and strategies used by adults with dyscalculia when performing fraction tasks. Exploring how symbolic fractions are processed by adults with dyscalculia might aid in understanding the misconceptions and challenges faced by them on fraction tasks. Our results show that the performance of adults with dyscalculia was lower for both symbolic and nonsymbolic tasks as compared to typical adults. Further, effective effortful strategies like componential processing were not utilized by adults with dyscalculia which might have been one of the reasons for a lower accuracy score. This indicates that adults with dyscalculia struggle with equivalent fractions and are unable to utilize componential processing strategies effectively as compared to typical adults.

\section{Conclusion}

The aim of this research was twofold; first, to examine perceptual sensitivity towards nonsymbolic ratio magnitudes in participants with varying levels of math skills and second, to investigate the extent to which the perceptual ability is utilized for symbolic fraction processing. We found evidence for perceptual sensitivity towards non-symbolic ratio magnitudes in all groups of participants, irrespective of their age or level of math skill. A clear pattern that also emerged in our study was that children and adults with and without dyscalculia do not rely on the RPS when they have to find equivalent symbolic fractions (Experiments 1 to 3). However, they do rely on the RPS when the ratios behind symbolic fractions have to be estimated (Experiment 


\section{Page 37 of 60 \\ RPS AND SYMBOLIC FRACTIONS}

4). Together these findings add to growing evidence for an intuitive, perceptual sensitivity for non-symbolic relational magnitudes. They also indicate that the role of the RPS in symbolic fraction processing is limited to tasks in which participants have to approximate the value of a fraction. 


\section{RPS AND SYMBOLIC FRACTIONS}

\section{Supplementary Material}

The Supplementary Material is available at: qjep.sagepub.com 


\section{References}

Alibali, M. W., \& Sidney, P. G. (2015). Variability in the natural number bias: Who, when, how, and why. Learning and Instruction, 36-61. https://doi.org/10.1016/j.learninstruc.2015.01.003

Bailey, D. H., Hoard, M. K., Nugent, L., \& Geary, D. C. (2012). Competence with fractions predicts gains in mathematics achievement. Journal of Experimental Child Psychology, 113(3), 447-455. https://doi.org/10.1016/j.jecp.2012.06.004

Bonato, M., Fabbri, S., Umiltà, C., \& Zorzi, M. (2007). The mental representation of numerical fractions: Real or integer? Journal of Experimental Psychology: Human Perception and Performance, 33(6), 1410-1419. https://doi.org/10.1037/0096-1523.33.6.1410

Booth, J. L., \& Newton, K. J. (2012). Fractions: Could they really be the gatekeeper's doorman? Contemporary Educational Psychology, 37(4), 247-253. https://doi.org/10.1016/j.cedpsych.2012.07.001

Boyer, T. W., Levine, S. C., \& Huttenlocher, J. (2008). Development of proportional reasoning: Where young children go wrong. Developmental Psychology, 44(5), 1478-1490. https://doi.org/10.1037/a0013110

Butterworth, B., 2005. Developmental dyscalculia. In: Campbell, J.I.D. (Ed.), Handbook of Mathematical Cognition. Psychology Press, Hove, pp. 455-467.

Butterworth, B. (2011). Foundational Numerical Capacities and the Origins of Dyscalculia. In Space, Time and Number in the Brain (pp. 249-265). Elsevier. https://doi.org/10.1016/B978-0-12-385948-8.00016-5

Rumbaugh, D. M., Washburn, D. A., Boysen, S. T., \& Capaldi, E. J. (1993). The development of numerical competence: Animal and human models. 
Braithwaite, D. W., \& Siegler, R. S. (2017). Developmental changes in the whole number bias. Developmental Science, 21(2), e12541. https://doi.org/10.1111/desc.12541

Brysbaert, M. (2019). How Many Participants Do We Have to Include in Properly Powered Experiments? A Tutorial of Power Analysis with Reference Tables. Journal of Cognition, 2(1). https://doi.org/10.5334/joc. 72

Carpenter, T. P., Kepner, H., Corbitt, M. K., Lindquist, M. M., \& Reys, R. E. (1980). Results and Implications of the Second NAEP Mathematics Assessments: Elementary School. The Arithmetic Teacher, 27(8), 10-12, 44-47. Retrieved from http://www.jstor.org/stable/41191725

Chan, W.-H., Leu, Y.-C., \& Chen, C.-M. (2007). Exploring Group-Wise Conceptual Deficiencies of Fractions for Fifth and Sixth Graders in Taiwan. The Journal of Experimental Education, 76(1), 26-57. https://doi.org/10.3200/JEXE.76.1.26-58

Clarke, D. M., \& Roche, A. (2009). Students' fraction comparison strategies as a window into robust understanding and possible pointers for instruction. Educational Studies in Mathematics, 72(1), 127-138. https://doi.org/10.1007/s10649-009-9198-9

Cowan, N. (2016). Working Memory Maturation: Can We Get at the Essence of Cognitive Growth? Perspectives on Psychological Science, 11(2), 239-264. https://doi.org/10.1177/1745691615621279

De Brauwer, J., Verguts, T., \& Fias, W. (2006). The representation of multiplication facts: Developmental changes in the problem size, five, and tie effects. Journal of Experimental Child Psychology, 94(1), 43-56. https://doi.org/10.1016/j.jecp.2005.11.004

Dehaene, S., \& Cohen, L. (2007). Cultural Recycling of Cortical Maps. Neuron, 56(2), 384-398. https://doi.org/10.1016/j.neuron.2007.10.004 


\section{RPS AND SYMBOLIC FRACTIONS}

Dehaene, S., Dehaene-Lambertz, G., \& Cohen, L. (1998a). Abstract representations of numbers in the animal and human brain. Trends in Neurosciences, 21(8), 355-361. https://doi.org/10.1016/S0166-2236(98)01263-6

Dehaene, S., Dehaene-Lambertz, G., \& Cohen, L. (1998b). Abstract representations of numbers in the animal and human brain. Trends in Neurosciences, 21(8), 355-361. https://doi.org/10.1016/S0166-2236(98)01263-6

De Smedt, B., Noël, M.-P., Gilmore, C., \& Ansari, D. (2013). How do symbolic and nonsymbolic numerical magnitude processing skills relate to individual differences in children's mathematical skills? A review of evidence from brain and behavior. Trends in Neuroscience and Education, 2(2), 48-55. https://doi.org/10.1016/j.tine.2013.06.001

Faulkenberry, T. J., \& Pierce, B. H. (2011). Mental Representations in Fraction Comparison: Holistic Versus Component-Based Strategies. Experimental Psychology, 58(6), 480-489. https://doi.org/10.1027/1618-3169/a000116

Feigenson, L., Dehaene, S., \& Spelke, E. (2004). Core systems of number. Trends in Cognitive Sciences, 8(7), 307-314. https://doi.org/10.1016/j.tics.2004.05.002

Fuchs, L. S., Schumacher, R. F., Sterba, S. K., Long, J., Namkung, J., Malone, A., ... Changas, P. (2014). Does working memory moderate the effects of fraction intervention? An aptitudetreatment interaction. Journal of Educational Psychology, 106(2), 499-514. https://doi.org/10.1037/a0034341

Gabriel, F., Coché, F., Szucs, D., Carette, V., Rey, B., \& Content, A. (2013). A componential view of children's difficulties in learning fractions. Frontiers in Psychology, 4. https://doi.org/10.3389/fpsyg.2013.00715 
Gelman, R., \& Meck, E. (1983). Preschoolers' counting: Principles before skill. Cognition, 13(3), 343-359. https://doi.org/10.1016/0010-0277(83)90014-8

Leibovich, T., Katzin, N., Harel, M., \& Henik, A. (2017). From "sense of number" to "sense of magnitude": The role of continuous magnitudes in numerical cognition. Behavioral and Brain Sciences, 40, e164. https://doi.org/10.1017/S0140525X16000960

Leslie, A. M., Gelman, R., \& Gallistel, C. R. (2008). The generative basis of natural number concepts. Trends in Cognitive Sciences, 12(6), 213-218. https://doi.org/10.1016/j.tics.2008.03.004

Lewis, M. R., Matthews, P. G., \& Hubbard, E. M. (2016). Neurocognitive architectures and the nonsymbolic foundations of fractions understanding. In Development of Mathematical Cognition (pp. 141-164). Academic Press.

González-Forte, J. M., Fernández, C., \& Dooren, W. V. (2019). EXPLORING STUDENTS' REASONING ABOUT FRACTION MAGNITUDE. 9.

Halberda, J., Mazzocco, M. M. M., \& Feigenson, L. (2008). Individual differences in non-verbal number acuity correlate with maths achievement. $455,5$.

Hansen, N., Rinne, L., Jordan, N. C., Ye, A., Resnick, I., \& Rodrigues, J. (2017). Codevelopment of fraction magnitude knowledge and mathematics achievement from fourth through sixth grade. Learning and Individual Differences, 60, 18-32. https://doi.org/10.1016/j.lindif.2017.10.005

Hecht, S. A., Close, L., \& Santisi, M. (2003). Sources of individual differences in fraction skills. Journal of Experimental Child Psychology, 86(4), 277-302. https://doi.org/10.1016/j.jecp.2003.08.003 


\section{RPS AND SYMBOLIC FRACTIONS}

Hurst, M. A., \& Cordes, S. (2018). Attending to relations: Proportional reasoning in 3- to 6-yearold children. Developmental Psychology, 54(3), 428-439. https://doi.org/10.1037/dev0000440

Hurst, M., \& Cordes, S. (2016). Rational-number comparison across notation: Fractions, decimals, and whole numbers. Journal of Experimental Psychology: Human Perception and Performance, 42(2), 281-293. https://doi.org/10.1037/xhp0000140

Imbo, I., \& Vandierendonck, A. (2008). Effects of problem size, operation, and working-memory span on simple-arithmetic strategies: Differences between children and adults? Psychological Research, 72(3), 331-346. https://doi.org/10.1007/s00426-007-0112-8

Ischebeck, A., Schocke, M., \& Delazer, M. (2009). The processing and representation of fractions within the brain. NeuroImage, 47(1), 403-413. https://doi.org/10.1016/j.neuroimage.2009.03.041

Iuculano, T., Tang, J., Hall, C. W. B., \& Butterworth, B. (2008). Core information processing deficits in developmental dyscalculia and low numeracy. Developmental Science, 11(5), 669-680. https://doi.org/10.1111/j.1467-7687.2008.00716.x

Jacob, S. N., \& Nieder, A. (2009). Notation-Independent Representation of Fractions in the Human Parietal Cortex. Journal of Neuroscience, 29(14), 4652-4657. https://doi.org/10.1523/JNEUROSCI.0651-09.2009

Jacob, Simon N., \& Nieder, A. (2009). Tuning to non-symbolic proportions in the human frontoparietal cortex: Representation of proportions in the human brain. European Journal of Neuroscience, 30(7), 1432-1442. https://doi.org/10.1111/j.14609568.2009.06932.x 
Jeong, Y., Levine, S. C., \& Huttenlocher, J. (2007). The Development of Proportional Reasoning: Effect of Continuous Versus Discrete Quantities. Journal of Cognition and Development, 8(2), 237-256. https://doi.org/10.1080/15248370701202471

Jordan, N. C., Hansen, N., Fuchs, L. S., Siegler, R. S., Gersten, R., \& Micklos, D. (2013). Developmental predictors of fraction concepts and procedures. Journal of Experimental Child Psychology, 116(1), 45-58. https://doi.org/10.1016/j.jecp.2013.02.001

Kallai, A. Y., \& Tzelgov, J. (2009). A generalized fraction: An entity smaller than one on the mental number line. Journal of Experimental Psychology: Human Perception and Performance, 35(6), 1845-1864. https://doi.org/10.1037/a0016892

Kallai, A. Y., \& Tzelgov, J. (2012). When meaningful components interrupt the processing of the whole: The case of fractions. Acta Psychologica, 139(2), 358-369. https://doi.org/10.1016/j.actpsy.2011.11.009

Kalra, P. B., Binzak, J. V., Matthews, P. G., \& Hubbard, E. M. (2020). Symbolic fractions elicit an analog magnitude representation in school-age children. Journal of Experimental Child Psychology, 195, 104844. https://doi.org/10.1016/j.jecp.2020.104844

Kieren, T. E. (1981). Five faces of mathematical knowledge building. Edmonton: Department of Secondary Education, University of Alberta.

Kloosterman, P. (2010). Mathematics Skills of 17-Year-Olds in the United States: 1978 to 2004. Journal for Research in Mathematics Education, 41(1), 20-51. Retrieved from http://www.jstor.org/stable/40539363

Leslie, A. M., Gelman, R., \& Gallistel, C. R. (2008). The generative basis of natural number concepts. Trends in Cognitive Sciences, 12(6), 213-218. https://doi.org/10.1016/j.tics.2008.03.004 


\section{RPS AND SYMBOLIC FRACTIONS}

Mack, N. K. (1990). Learning Fractions with Understanding: Building on Informal Knowledge. Journal for Research in Mathematics Education, 16. https://doi.org/10.2307/749454

Matthews, P. G. (2015, March). Delimiting and leveraging children's natural sense of proportion. Poster presented at the Biennial Meeting of the Society for Research in Child Development (SRCD), Philadelphia, PA

Matthews, P. G., \& Chesney, D. L. (2015). Fractions as percepts? Exploring cross-format distance effects for fractional magnitudes. Cognitive Psychology, 78, 28-56. https://doi.org/10.1016/j.cogpsych.2015.01.006

Matthews, P. G., \& Ellis, A. B. (2018). Natural alternatives to natural number: The case of ratio. Journal of Numerical Cognition, 4(1), 19-58.

Matthews, P. G., Chesney, D. L., \& McNeil, N. M. (n.d.). Are Fractions Natural Numbers, Too? 6.

Matthews, P. G., Lewis, M. R., \& Hubbard, E. M. (2016). Individual Differences in Nonsymbolic Ratio Processing Predict Symbolic Math Performance. Psychological Science, 27(2), 191-202. https://doi.org/10.1177/0956797615617799

Matthews, P. G., \& Ziols, R. (2019). What's Perception Got To Do with It? Re-framing Foundations for Rational Number Concepts. In A. Norton \& M. W. Alibali (Eds.), Constructing Number (pp. 213-235). https://doi.org/10.1007/978-3-030-00491-0_10

Mazzocco, M. M. M., \& Devlin, K. T. (2008). Parts and 'holes': Gaps in rational number sense among children with vs. without mathematical learning disabilities. Developmental Science, 11(5), 681-691. https://doi.org/10.1111/j.1467-7687.2008.00717.x 
RPS AND SYMBOLIC FRACTIONS

Mazzocco, M. M. M., Feigenson, L., \& Halberda, J. (2011). Impaired Acuity of the Approximate Number System Underlies Mathematical Learning Disability (Dyscalculia): Impaired Numerical Acuity Contributes to MLD. Child Development, 82(4), 1224-1237. https://doi.org/10.1111/j.1467-8624.2011.01608.x

Mazzocco, M. M. M., Myers, G. F., Lewis, K. E., Hanich, L. B., \& Murphy, M. M. (2013). Limited knowledge of fraction representations differentiates middle school students with mathematics learning disability (dyscalculia) versus low mathematics achievement. Journal of Experimental Child Psychology, 115(2), 371-387. https://doi.org/10.1016/j.jecp.2013.01.005

McCrink, K., Spelke, E. S., Dehaene, S., \& Pica, P. (2013). Non-symbolic halving in an Amazonian indigene group. Developmental Science, 16(3), 451-462. https://doi.org/10.1111/desc. 12037

McCrink, K., \& Wynn, K. (2007). Ratio Abstraction by 6-Month-Old Infants. Psychological Science, 18(8), 740-745. https://doi.org/10.1111/j.1467-9280.2007.01969.x

Meert, G., Grégoire, J., \& Noël, M.-P. (2009). Rational numbers: Componential versus holistic representation of fractions in a magnitude comparison task. Quarterly Journal of $\begin{array}{lll}\text { Experimental Psychology, } & \text { 62(8), }\end{array}$ https://doi.org/10.1080/17470210802511162

Meert, G., Grégoire, J., \& Noël, M.-P. (2010). Comparing 5/7 and 2/9: Adults can do it by accessing the magnitude of the whole fractions. Acta Psychologica, 135(3), 284-292. https://doi.org/10.1016/j.actpsy.2010.07.014

Meert, G., Grégoire, J., Seron, X., \& Noël, M.-P. (2012). The mental representation of the magnitude of symbolic and nonsymbolic ratios in adults. Quarterly Journal of 
RPS AND SYMBOLIC FRACTIONS

$\begin{array}{lll}\text { Experimental Psychology, } & \text { 65(4), }\end{array}$ https://doi.org/10.1080/17470218.2011.632485

Meert, G., Grégoire, J., Seron, X., \& Noël, M.-P. (2013). The Processing of Symbolic and Nonsymbolic Ratios in School-Age Children. PLoS ONE, 8(11), e82002. https://doi.org/10.1371/journal.pone.0082002

Mix, K. S., Levine, S. C., \& Huttenlocher, J. (1999). Early Fraction Calculation Ability. 11.

Möhring, W., Newcombe, N. S., Levine, S. C., \& Frick, A. (2016). Spatial Proportional Reasoning Is Associated With Formal Knowledge About Fractions. Journal of Cognition and Development, 17(1), 67-84. https://doi.org/10.1080/15248372.2014.996289

Moyer, R. S., \& Landauer, T. K. (1967). Time required for judgements of numerical inequality. Ni, Y. (2001). Semantic Domains of Rational Numbers and the Acquisition of Fraction Equivalence. Contemporary Educational Psychology, 26(3), 400-417. https://doi.org/10.1006/ceps.2000.1072

Ni, Y., \& Zhou, Y.-D. (2005). Teaching and Learning Fraction and Rational Numbers: The Origins and Implications of Whole Number Bias. Educational Psychologist, 40(1), 27-52. https://doi.org/10.1207/s15326985ep4001_3

Obersteiner, A., Van Dooren, W., Van Hoof, J., \& Verschaffel, L. (2013). The natural number bias and magnitude representation in fraction comparison by expert mathematicians. Learning and Instruction, 28, 64-72. https://doi.org/10.1016/j.learninstruc.2013.05.003

Piazza, M. (2010). Neurocognitive start-up tools for symbolic number representations. Trends in Cognitive Sciences, 14(12), 542-551. https://doi.org/10.1016/j.tics.2010.09.008

Piazza, M., Facoetti, A., Trussardi, A. N., Berteletti, I., Conte, S., Lucangeli, D., ... Zorzi, M. (2010). Developmental trajectory of number acuity reveals a severe impairment in 
RPS AND SYMBOLIC FRACTIONS
developmental
dyscalculia.
Cognition,
$116(1)$
33-41. https://doi.org/10.1016/j.cognition.2010.03.012

Pica, P. (2004). Exact and Approximate Arithmetic in an Amazonian Indigene Group. Science, 306(5695), 499-503. https://doi.org/10.1126/science.1102085

Pitkethly, A., \& Hunting, R. (1996). A review of recent research in the area of initial fraction concepts. Educational Studies in Mathematics, 30(1), 5-38 https://doi.org/10.1007/BF00163751

Post, T., Behr, M. J., \& Lesh, R. (1986). Research-based observations about children's learning of rational number concepts. Focus on Learning Problems in Mathematics, 8(1), 39-48.

Price, G. R., \& Ansari, D. (2013). Dyscalculia: Characteristics, causes, and treatments. Numeracy, 6(1), 2.

Rodrigues, J., \& Jordan, N. C. (2019). Identifying Fraction Measures as Screeners of Mathematics Risk Status. Journal of Learning Disabilities, 18.

Schneider, M., \& Siegler, R. S. (2010). Representations of the magnitudes of fractions. Journal of Experimental Psychology: Human Perception and Performance, 36(5), 1227-1238. https://doi.org/10.1037/a0018170

Siegler, R. S., Duncan, G. J., Davis-Kean, P. E., Duckworth, K., Claessens, A., Engel, M., ... Chen, M. (2012). Early Predictors of High School Mathematics Achievement. Psychological Science, 23(7), 691-697. https://doi.org/10.1177/0956797612440101

Siegler, R. S., Fazio, L. K., Bailey, D. H., \& Zhou, X. (2013). Fractions: The new frontier for theories of numerical development. Trends in Cognitive Sciences, 17(1), 13-19. https://doi.org/10.1016/j.tics.2012.11.004 


\section{RPS AND SYMBOLIC FRACTIONS}

Siegler, R. S., \& Pyke, A. A. (2013). Developmental and individual differences in understanding of fractions. Developmental Psychology, 49(10), 1994-2004. https://doi.org/10.1037/a0031200

Siegler, R. S., Thompson, C. A., \& Schneider, M. (2011). An integrated theory of whole number and fractions development. Cognitive Psychology, 62(4), 273-296. https://doi.org/10.1016/j.cogpsych.2011.03.001

Sophian, C. (2000). Perceptions of proportionality in young children: Matching spatial ratios. Cognition, 75(2), 145-170. https://doi.org/10.1016/S0010-0277(00)00062-7

Stafylidou, S., \& Vosniadou, S. (2004). The development of students' understanding of the numerical value of fractions. Learning and Instruction, 14(5), 503-518. https://doi.org/10.1016/j.learninstruc.2004.06.015

Steffe, L. P., \& Olive, J. (2010). Children's Fractional Knowledge. Springer US. https://doi.org/10.1007/978-1-4419-0591-8

Stigler, J. W., Givvin, K. B., \& Thompson, B. J. (2010). What Community College Developmental Mathematics Students Understand. 44.

Streefland, L. (1991). Fractions in realistic mathematics education: A paradigm of developmental research (Vol. 8). Springer Science \& Business Media.

Vallentin, D., \& Nieder, A. (2010). Representations of visual proportions in the primate posterior parietal and prefrontal cortices: Proportion processing in the monkey brain. European Journal of Neuroscience, 32(8), 1380-1387. https://doi.org/10.1111/j.14609568.2010.07427.x

Vamvakoussi, X., \& Vosniadou, S. (2010). How Many Decimals Are There Between Two Fractions? Aspects of Secondary School Students' Understanding of Rational Numbers 


\section{RPS AND SYMBOLIC FRACTIONS}

and Their Notation. Cognition and Instruction, 28(2), 181-209. https://doi.org/10.1080/07370001003676603

Van Hoof, J., Lijnen, T., Verschaffel, L., \& Van Dooren, W. (2013). Are secondary school students still hampered by the natural number bias? A reaction time study on fraction comparison tasks. Research in Mathematics Education, 15(2), 154-164. https://doi.org/10.1080/14794802.2013.797747

Yoshida, H., \& Sawano, K. (2002). Overcoming cognitive obstacles in learning fractions: Equalpartitioning and equal-whole1. Japanese Psychological Research, 44(4), 183-195. https://doi.org/10.1111/1468-5884.00021

Zhang, L., Fang, Q., Gabriel, F. C., \& Szucs, D. (2014). The componential processing of fractions in adults and children: Effects of stimuli variability and contextual interference. Frontiers in Psychology, 5. https://doi.org/10.3389/fpsyg.2014.00981 
RPS AND SYMBOLIC FRACTIONS

\section{Figure Legends}

Figure 1. Match-to-sample task. (A) In each non-symbolic trial, participants had to match the pair of line segments on the left of the screen to the equivalent pair of line segments on the right of the screen. (B) In each symbolic trial, participants had to match the fraction on the left of the screen to the equivalent fraction on the right of the screen.

Figure 2. Results of Experiment 1 (typical adults). (A) Proportion correct as a function of Notation and RoR for trials in which the numerator of the target was identical to the numerator of the distractor and in which the ratio between numerators of match and distractor was small. (B) Proportion correct as a function of Notation and RoR for trials in which the numerator of the target was identical from the numerator of the distractor and in which the ratio between numerators of match and distractor was large. (C) Proportion correct as a function of Notation and RoR for trials in which the numerator of the target was different from the numerator of the distractor and in which the ratio between numerators of match and distractor was small. (D) Proportion correct as a function of Notation and RoR for trials in which the numerator of the target was different from the numerator of the distractor and in which the ratio between numerators of match and distractor was large. The bars here represent standard errors.

Figure 3. Results of Experiment 2 (children). (A) Proportion correct as a function of Notation and RoR for trials in which the numerator of the target was identical to the numerator of the distractor and in which the ratio between numerators of match and distractor was small. (B) Proportion correct as a function of Notation and RoR for trials in which the numerator of the target was identical from the numerator of the distractor and in which the ratio between 
numerators of match and distractor was large. (C) Proportion correct as a function of Notation and RoR for trials in which the numerator of the target was different from the numerator of the distractor and in which the ratio between numerators of match and distractor was small. (D) Proportion correct as a function of Notation and RoR for trials in which the numerator of the target was different from the numerator of the distractor and in which the ratio between numerators of match and distractor was large. The bars here represent standard errors.

Figure 4. Results of Experiment 3 (adults with dyscalculia). (A) Proportion correct as a function of Notation and RoR for trials in which the numerator of the target was identical to the numerator of the distractor and in which the ratio between numerators of match and distractor was small. (B) Proportion correct as a function of Notation and RoR for trials in which the numerator of the target was identical from the numerator of the distractor and in which the ratio between numerators of match and distractor was large. (C) Proportion correct as a function of Notation and RoR for trials in which the numerator of the target was different from the numerator of the distractor and in which the ratio between numerators of match and distractor was small. (D) Proportion correct as a function of Notation and RoR for trials in which the numerator of the target was different from the numerator of the distractor and in which the ratio between numerators of match and distractor was large. The bars here represent standard errors.

Figure 5. Match-to-sample task in mixed-notation condition. In each trial, participants had to match the pair of line segments on the left of the screen to the equivalent fraction on the right side of the screen. 


\section{Page 53 of 60 \\ RPS AND SYMBOLIC FRACTIONS}

Figure 6. Results of Experiment 4 (adults, mixed-notation paradigm). Proportion correct as a function of RoR. The bars here represent standard errors. 
A.

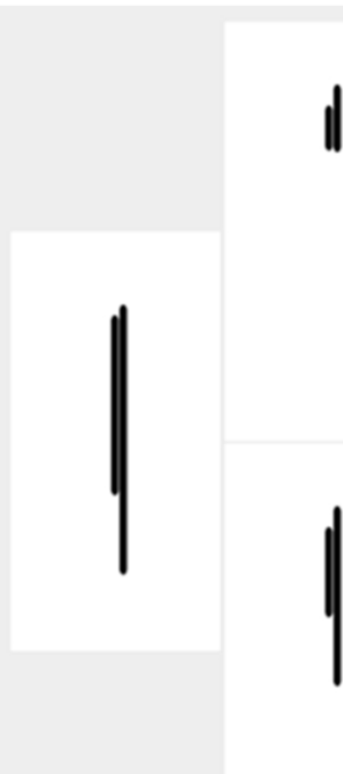

B.

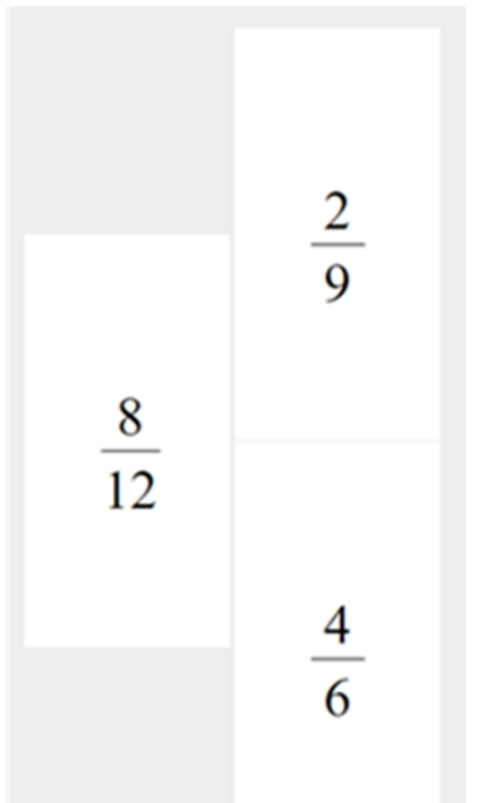

Figure 1. Match-to-sample task. (A) In each non-symbolic trial, participants had to match the pair of line segments on the left of the screen to the equivalent pair of line segments on the right of the screen. (B) In each symbolic trial, participants had to match the fraction on the left of the screen to the equivalent fraction on the right of the screen.

$254 \times 190 \mathrm{~mm}(600 \times 600 \mathrm{DPI})$ 

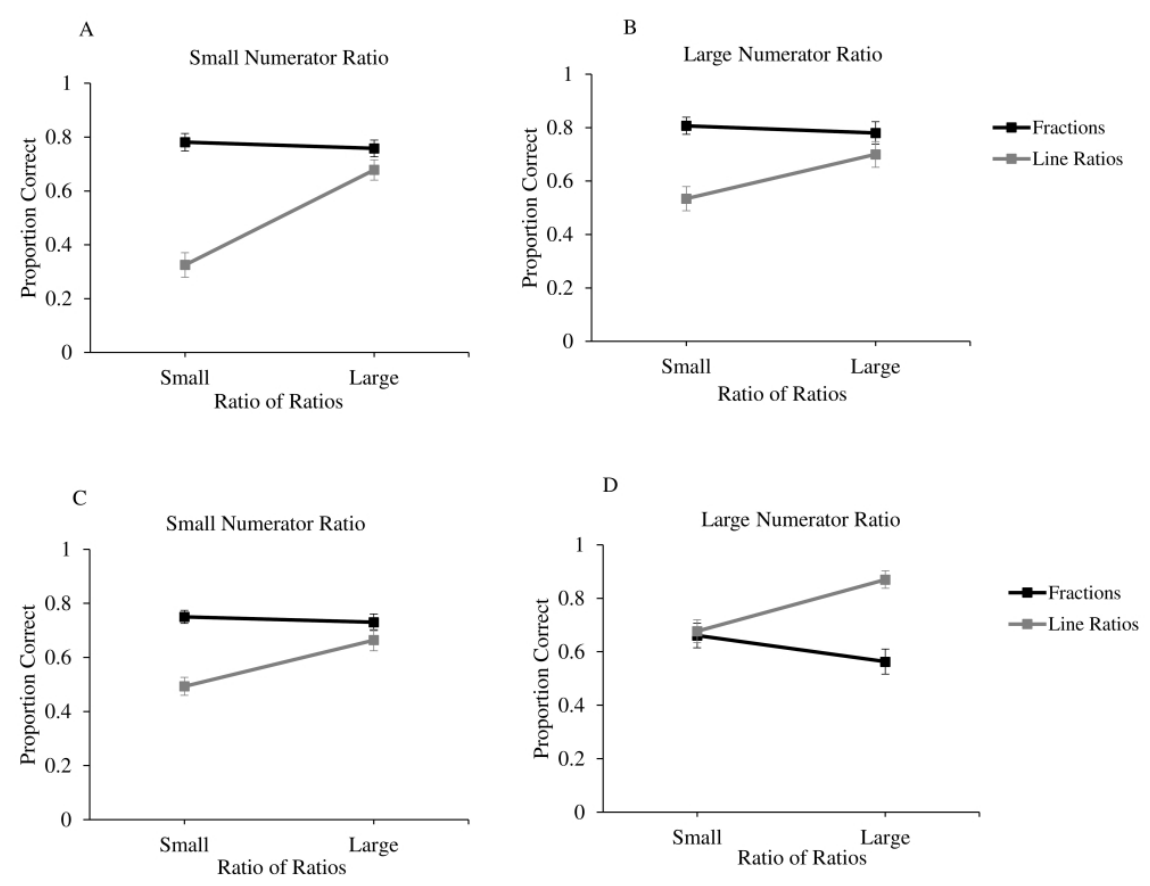

Figure 2. Results of Experiment 1 (typical adults). (A) Proportion correct as a function of Notation and RoR for trials in which the numerator of the target was identical to the numerator of the distractor and in which the ratio between numerators of match and distractor was small. (B) Proportion correct as a function of Notation and RoR for trials in which the numerator of the target was identical from the numerator of the distractor and in which the ratio between numerators of match and distractor was large. (C) Proportion correct as a function of Notation and RoR for trials in which the numerator of the target was different from the numerator of the distractor and in which the ratio between numerators of match and distractor was small. (D) Proportion correct as a function of Notation and RoR for trials in which the numerator of the target was different from the numerator of the distractor and in which the ratio between numerators of match and distractor was large. The bars here represent standard errors.

\section{$1611 \times 1173 \mathrm{~mm}(96 \times 96 \mathrm{DPI})$}



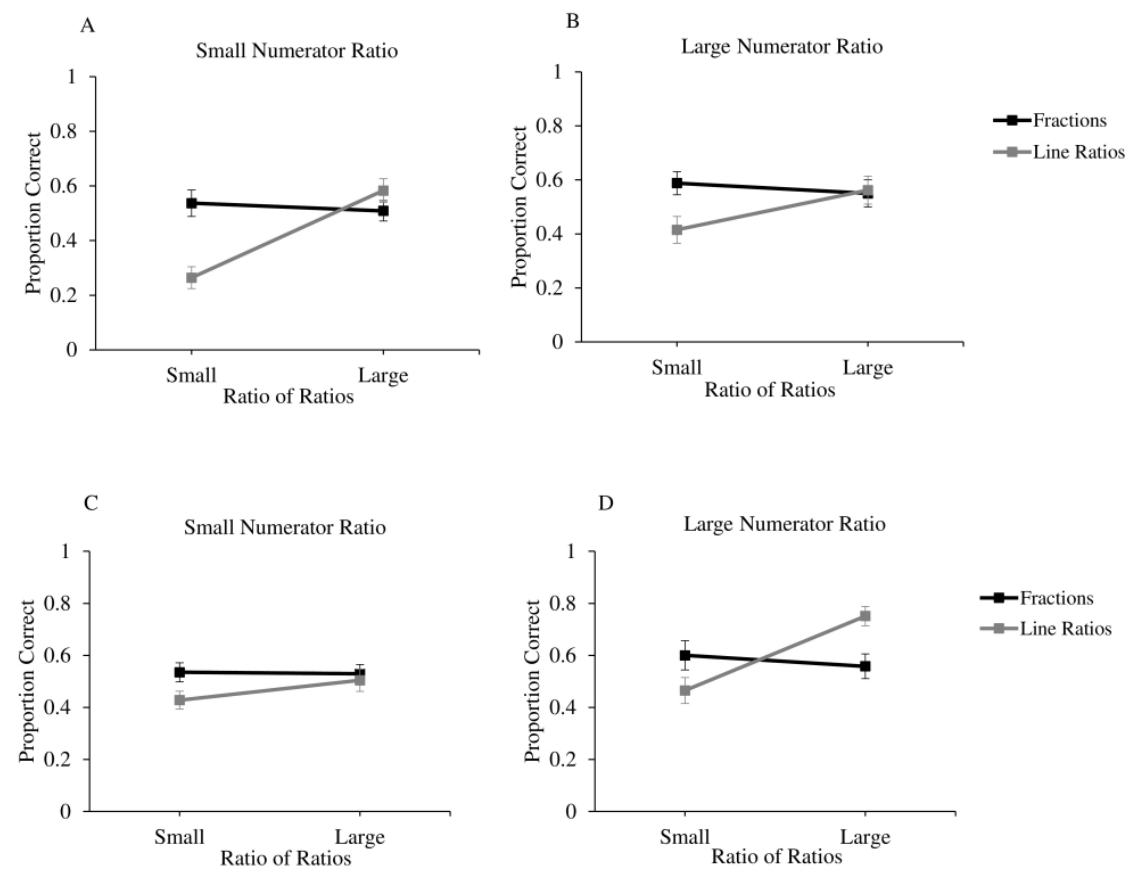

Figure 3. Results of Experiment 2 (children). (A) Proportion correct as a function of Notation and RoR for trials in which the numerator of the target was identical to the numerator of the distractor and in which the ratio between numerators of match and distractor was small. (B) Proportion correct as a function of Notation and RoR for trials in which the numerator of the target was identical from the numerator of the distractor and in which the ratio between numerators of match and distractor was large. (C) Proportion correct as a function of Notation and RoR for trials in which the numerator of the target was different from the numerator of the distractor and in which the ratio between numerators of match and distractor was small. (D) Proportion correct as a function of Notation and RoR for trials in which the numerator of the target was different from the numerator of the distractor and in which the ratio between numerators of match and distractor was large. The bars here represent standard errors.

$1588 \times 1155 \mathrm{~mm}(96 \times 96 \mathrm{DPI})$ 

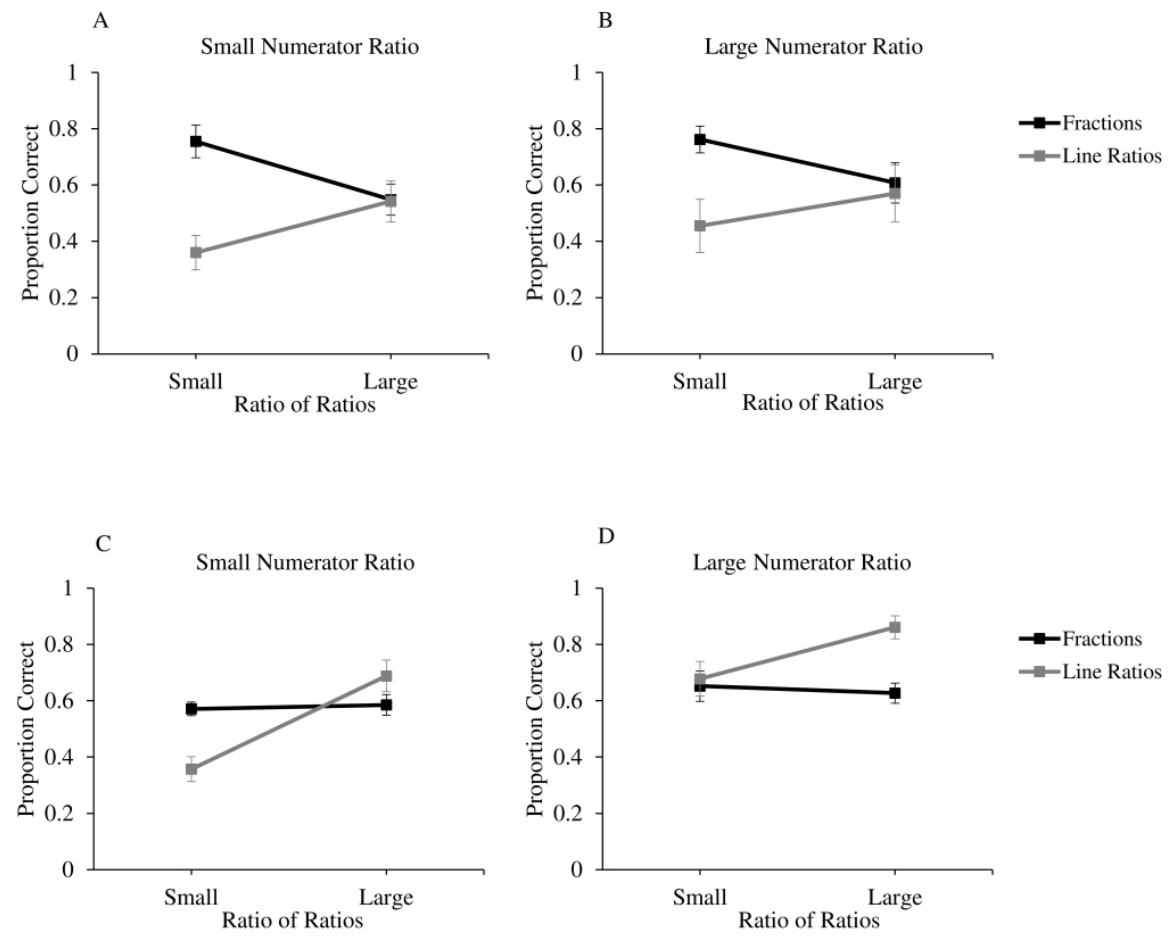

Figure 4. Results of Experiment 3 (adults with dyscalculia). (A) Proportion correct as a function of Notation and RoR for trials in which the numerator of the target was identical to the numerator of the distractor and in which the ratio between numerators of match and distractor was small. (B) Proportion correct as a function of Notation and RoR for trials in which the numerator of the target was identical from the numerator of the distractor and in which the ratio between numerators of match and distractor was large. (C) Proportion correct as a function of Notation and RoR for trials in which the numerator of the target was different from the numerator of the distractor and in which the ratio between numerators of match and distractor was small. (D) Proportion correct as a function of Notation and RoR for trials in which the numerator of the target was different from the numerator of the distractor and in which the ratio between numerators of match and distractor was large. The bars here represent standard errors. 
Figure 5. Match-to-sample task in mixed-notation condition. In each trial, participants had to match the pair of line segments on the left of the screen to the equivalent fraction on the right side of the screen.

\section{$254 \times 190 \mathrm{~mm}(600 \times 600 \mathrm{DPI})$}


Figure 6. Results of Experiment 4 (adults, mixed-notation paradigm). Proportion correct as a function of RoR. The bars here represent standard errors.

\section{$254 \times 190 \mathrm{~mm}(600 \times 600 \mathrm{DPI})$}

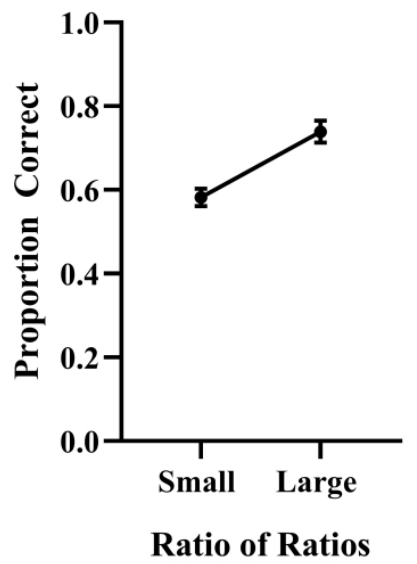


Table 1. Psychometric measures of adults with dyscalculia

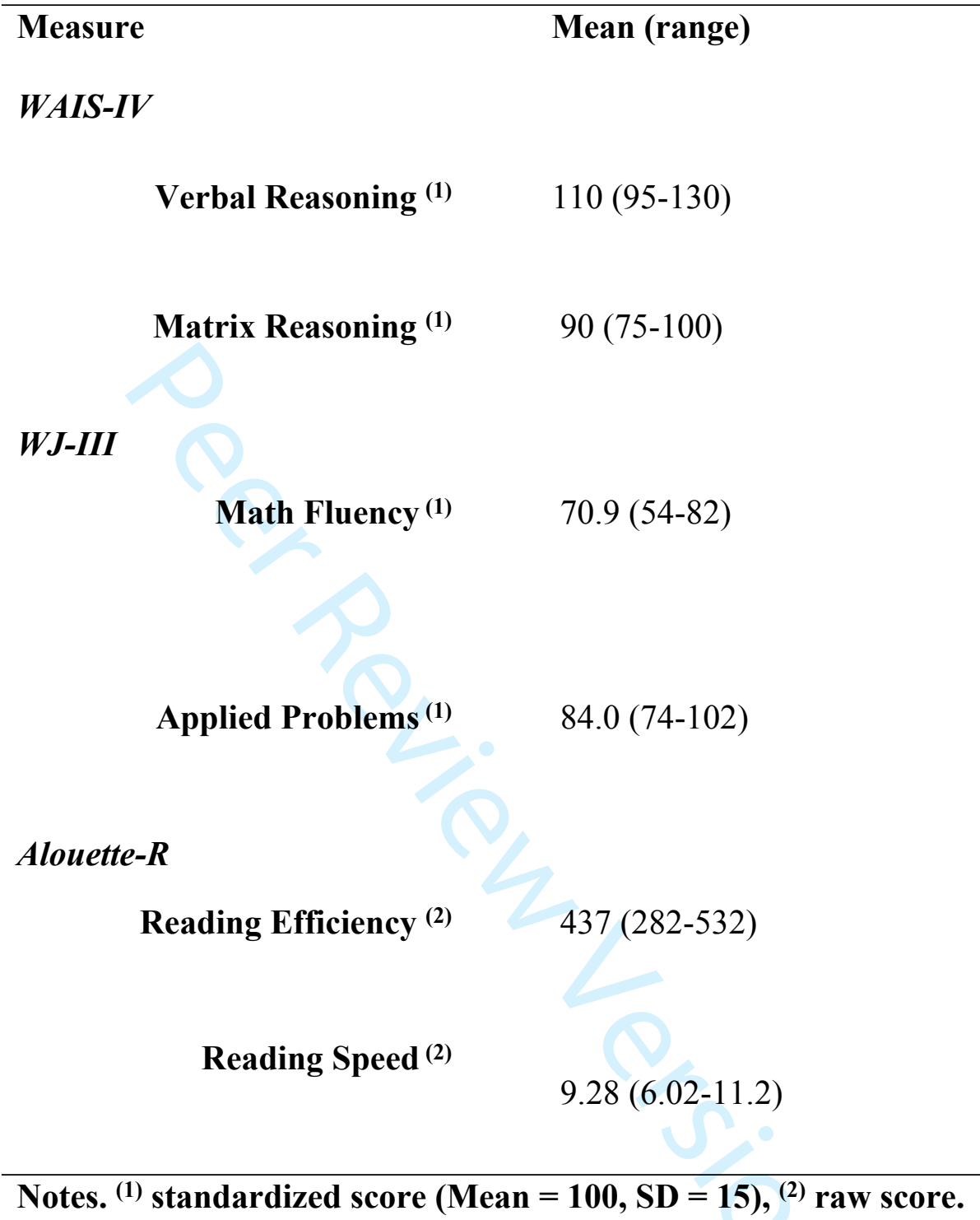

